DEPARTMENT OF ECONOMICS

Working Paper Series

Monetary Policy, Asset Prices, and Liquidity in Over-the-CounterMarkets

Athanasios Geromichalos

UC Davis

Lucas Herrenbrueck

UC Davis

September 25, 2012

Paper \# 12-20

We revisit a traditional topic in monetary economics: the relationship between asset prices and monetary policy. We study a model in which money helps facilitate trade in decentralized markets, as in Lagos andWright (2005), and real assets are traded in an over-the-counter (OTC) market, as in Duffie, G^arleanu, and Pedersen (2005). Agents wish to hold liquid portfolios, but liquidity comes at a cost: inflation. The OTC market serves as a secondary asset market, in which agents can rebalance their positions depending on their liquidity needs. Hence, a contribution of our paper is to provide a micro-founded explanation of the assumption that different investors have different valuations for the same asset, which is the key for generating gains from trade in the Duffie et al framework. In equilibrium, assets can be priced higher than their fundamental value because they help agents avoid the inflation tax.

Department of Economics One Shields Avenue

Davis, CA 95616

(530)752-0741

http://www.econ.ucdavis.edu/working_search.cfm 


\section{Monetary Policy, Asset Prices, and Liquidity in Over-the-Counter Markets}

\section{Athanasios Geromichalos}

University of California - Davis

\section{Lucas Herrenbrueck}

University of California - Davis

This Version: September 2012

PRELIMINARY AND INCOMPLETE - PLEASE DO NOT CIRCULATE

ABSTRACT

We revisit a traditional topic in monetary economics: the relationship between asset prices and monetary policy. We study a model in which money helps facilitate trade in decentralized markets, as in Lagos and Wright (2005), and real assets are traded in an over-the-counter (OTC) market, as in Duffie, Gârleanu, and Pedersen (2005). Agents wish to hold liquid portfolios, but liquidity comes at a cost: inflation. The OTC market serves as a secondary asset market, in which agents can rebalance their positions depending on their liquidity needs. Hence, a contribution of our paper is to provide a micro-founded explanation of the assumption that different investors have different valuations for the same asset, which is the key for generating gains from trade in the Duffie et al framework. In equilibrium, assets can be priced higher than their fundamental value because they help agents avoid the inflation tax.

JEL Classification: E31, E50, E52, G12

Keywords: monetary-search models, liquidity, asset prices, over-the-counter markets

Email: ageromich@ucdavis.edu, herrenbrueck@ucdavis.edu.

We are grateful to Jonathan Chiu, Miguel Molico, Guillaume Rocheteau, Christopher Waller, and Randall Wright for useful comments and suggestions. We would also like to thank participants at the Chicago Fed workshop on Money, Banking, Payments, and Finance, and at the Bank of Canada and UC Davis seminars for their feedback. 


\section{Introduction}

Since Tobin's (1965) influential work, the effect of monetary policy on asset prices has been a major topic of study in monetary economics. In the majority of papers that have researched this question, the assets under consideration are assumed to trade in perfectly competitive markets. However, a recent branch of the finance literature, following Duffie, Gârleanu, and Pedersen (2005) (DGP henceforth), has documented that many assets are in fact traded in overthe-counter (OTC) markets, characterized by search and bargaining frictions. In this paper, we revisit the traditional question of the effect of monetary policy on asset prices, within an environment in which assets can be traded in an OTC fashion.

We investigate this question using a model in the tradition of modern monetary-search theory, in which money serves as a medium of exchange in decentralized trade, and agents have periodical access to a centralized or Walrasian market, as in Lagos and Wright (2005). A real asset in fixed supply is first traded (issued) in the centralized market, where agents also choose their money holdings. After learning whether they will have an opportunity to consume in the decentralized market, agents may visit a secondary asset market where they can rebalance their positions depending on their liquidity needs. This secondary asset market resembles the frictional market of DGP, in which trade is bilateral and agents bargain over the price, allowing us to link asset pricing in OTC markets to monetary policy. Since the primary asset market is competitive, we can also study the effect of monetary policy on the competitive issue price, and compare our findings to the those of the existing literature.

In standard asset pricing theory, the price of the asset would reflect only the discounted stream of dividends (the fundamental value), since the asset serves only as a store of value. However, in our model, the real asset provides another service: owners of the asset can swap it with money in the OTC market, after the uncertainty regarding consumption in the decentralized market has been resolved. Thus, the asset helps agents avoid the inflation tax. We show that, if the supply of the asset is sufficiently small, the issue price of the asset can exceed the fundamental value: the asset carries a liquidity premium. As inflation rises, this premium increases because the service that the asset provides, avoiding the inflation tax, becomes more essential.

Although the effect of inflation on the primary asset price is generally positive and can be described in a straightforward way, its effect on the secondary asset price is ambiguous. In principle, higher inflation generates two opposing forces. Ex ante, all agents carry less money, which increases the value of additional liquidity for agents who get a consumption opportunity (sellers of assets in the OTC). These agents are willing to accept a lower price for their assets. On the other hand, the higher inflation tax reduces the value of money, which makes agents without a consumption opportunity (buyers of assets in the OTC) willing to pay a higher price per unit of asset. Which force dominates depends on the bargaining power and on how sensitive real balances are to inflation. 
Since in our model the real asset is perfectly divisible, we can also examine the effect of inflation on the volume of OTC trade. We find that this variable is hump-shaped. Intuitively, when inflation is low, agents enter the OTC market with a lot of money, which diminishes their need for additional money, implying that only a small volume of assets will be traded. As inflation increases, agents carry less money, and the volume of trade in the OTC market rises. However, if inflation increases further, the trade volume declines, not because the need of agents to avoid the inflation tax is not strong enough, but because there is not enough liquidity in the OTC to facilitate asset trade.

One of the main contributions of this paper is to extend the DGP framework in two important ways. First, in the model of DGP, gains from trade arise from the assumption that agents have different valuations for the same asset. In fact, DGP motivate this assumption by arguing that a seller of the asset has a high need for liquidity. Our model formalizes precisely this idea: all participants in the OTC market have the same valuation for the asset, but sellers of the asset are agents who have a consumption opportunity and, hence, a high need for liquidity. In this way, our paper provides a micro-founded explanation for a key assumption of the DGP model. Second, agents in the DGP model have access to a risk-free real bank account which serves as a liquid asset and ensures that all efficient trades will be consummated. In our model, liquidity comes at a cost, inflation. This provides a channel through which monetary policy can affect the terms of trade in OTC asset markets. For instance, in a regime of high inflation, trade may be rationed due to a lack of liquidity, a result that could not be obtained in the DGP framework.

Another contribution of our paper is to provide a framework that intergrates the different definitions of asset liquidity in monetary economics and finance. As Lagos (2008) points out, "there seems to be $[\ldots]$ no definition of 'liquidity' that is generally agreed upon". In monetary theory, liquidity is an attribute of an asset, and it refers to how easily the asset can be transformed into consumption. In finance, on the other hand, liquidity is an attribute of a market, and it refers to how easily an investor can find a counterparty for trade, and at what cost. ${ }^{1}$ Our model bridges this gap: the frictions associated with search and bargaining in the OTC market determine how easily the asset can be transformed into the medium of exchange and, hence, into consumption.

Other papers in recent literature also introduce a real asset in the monetary-search framework and find that the asset price can include a liquidity premium, which is increasing in inflation. Prominent examples are Geromichalos, Licari, and Suarez-Lledo (2007), Lagos (2011), Lester, Postlewaite, and Wright (2012), and Jacquet and Tan (2010). However, it should be pointed out that the mechanism leading to these results is quite different in our model. In the papers listed above, the asset carries a liquidity premium because it competes with money as a medium of exchange. An increase in inflation induces agents to substitute money with

\footnotetext{
${ }^{1}$ A representative example from the monetary-search literature is Lagos and Wright (2005). For the financerelated definition, see DGP or Lagos and Rocheteau (2009).
} 
the asset, thus increasing the demand for the asset and, consequently, its price. In our model, the asset carries a premium because it can help agents increase their liquidity indirectly, when swapped with money in the OTC market. As inflation increases, money is still the only means of payment, but agents can use the asset to redistribute their money holdings more efficiently. Providing this service is what makes the asset more valuable in equilibrium.

Another feature that distinguishes our model from the related literature is that the asset carries a liquidity premium only for intermediate levels of inflation. If inflation is very low, agents carry enough money so that an additional unit of the asset does not help them obtain essential liquidity in the OTC market. On the other extreme, if inflation is very high, agents carry so little money that even a small supply of the asset can be enough to acquire all the available real balances, and the marginal unit of the asset is only useful as a store of value. In fact, because money is the only medium of exchange, a monetary equilibrium exists even for very high levels of inflation. In contrast, in papers that allow the asset to serve as a medium of exchange, if inflation rises beyond a certain point, monetary equilibrium ceases to exist.

Some recent papers exploit the idea that assets can carry liquidity premia and offer a new perspective for looking at long-standing asset-related puzzles. For example, Lagos (2010) develops an asset-pricing model in which assets are useful in facilitating exchange, and studies the implications of this channel for the equity premium puzzle and the risk-free rate puzzle. Geromichalos and Simonovska (2011) employ a related framework to examine the asset home bias puzzle. In these papers, assets serve as media of exchange, an assumption which might be subject to criticism as, typically, we do not observe agents trade real assets directly for goods. Lagos (2011) argues that assets can carry liquidity premia in broader contexts: for example, they may serve as collateral or help agents enter a repurchase agreement. ${ }^{2}$ We view our analysis as taking this argument a step further. The key liquidity property of an asset in our model is that it can be sold before maturity, in exchange for money, and can therefore facilitate trade without itself serving as a medium of exchange, as collateral, or in a repurchase agreement.

Our paper is also related to a strand of the monetary theory literature where agents get an opportunity to rebalance their money holdings when a consumption opportunity arises. Kocherlakota (2003) shows that nominal bonds enable agents to engage in intertemporal exchanges of money only if they are illiquid (costly to exchange for goods). Boel and Camera (2006) study how agents can use nominal bonds to insure against heterogeneous consumption risk, and find that the Friedman rule cannot implement the constrained-efficient allocation. Berentsen, Camera, and Waller (2007) introduce competitive banks that help allocate real balances into the hands of agents who currently have a consumption opportunity. They show that gains in welfare stem from the payment of interest on idle deposits and not from relaxing borrowers' liquidity constraints. Berentsen and Waller (2011) compare economies with borrowing

\footnotetext{
${ }^{2}$ For example, $\mathrm{Li}$ and $\mathrm{Li}$ (2010) study the liquidity properties of assets which can be used as collateral to secure loans, within an environment in which lenders cannot force borrowers to repay their debt.
} 
(inside bonds) to economies with selling assets (outside bonds). They find that allocations in the first economy can be replicated in the second economy, but under optimal policy, the allocations are equivalent. There are two key differences between our paper and this strand of the literature. First, we consider the case of a real asset, which allows us to link our results to traditional asset pricing theory. Second, we model the secondary asset market as an OTC market $a$ la DGP, which allows us to study the effect of monetary policy on asset pricing under frictions.

Finally, our paper is related to a growing literature in finance that builds upon the DGP framework in order to answer a variety of interesting questions regarding asset trade in markets with search frictions. For example, Vayanos and Weill (2006) provide a search based theory of the on-the-run phenomenon, Lagos, Rocheteau, and Weill (2011) consider crises and recoveries in OTC markets, and Chiu and Koeppl (2011) study the trading dynamics in OTC markets where the quality of assets is private information. We view our paper as a contribution to this literature because it provides a framework which links trade in OTC markets with monetary policy. Hence, our model can be used to examine whether monetary policy can affect the answer to these questions, and how.

The remainder of the paper is organized as follows. Section 2 describes the physical environment. Section 3 analyzes the terms of trade in the OTC market and the optimal behavior of agents. Section 4 describes a steady state equilibrium and examines the effects of monetary policy on asset prices and welfare. Section 5 concludes.

\section{Physical Environment}

Time is discrete with an infinite horizon, and each period consists of three sub-periods. During the first sub-period, economic activity takes place in a traditional Walrasian or centralized market. We refer to this market as the CM. In the second sub-period, a secondary asset market opens, which resembles the Over-the-Counter markets of DGP. We refer to this market as the OTC market. In the third sub-period agents visit a decentralized market for goods, similar to Lagos and Wright (2005), where bilateral and anonymous trade takes place. We term this the LW market. A detailed description of these markets will follow. There are two types of agents, buyers and sellers, depending on their role in the LW market. All agents live forever and their types are permanent. The measure of buyers is normalized to the unit. The measure of sellers will not be crucial for any of the results (there is no free entry), and it is discussed below.

All agents discount the future between periods (but not sub-periods) at rate $\beta \in(0,1)$. Buyers consume in the first and the third sub-periods and supply labor in the first sub-period. Their preferences for consumption and labor within a period are given by $\mathcal{U}(X, H, q)$, where $X, H$ represent consumption and labor in the $\mathrm{CM}$, respectively, and $q$ consumption in the LW market. Sellers consume only in the CM, and they produce in both the CM and the LW market. Their 
preferences are given by $\mathcal{V}(X, H, h)$, where $X, H$ are as above, and $h$ stands for hours worked in the LW market. Following Lagos and Wright (2005), we adopt the functional forms

$$
\begin{aligned}
& \mathcal{U}(X, H, q)=U(X)-H+u(q), \\
& \mathcal{V}(X, H, h)=U(X)-H-c(h) .
\end{aligned}
$$

We assume that $u$ and $U$ are twice continuously differentiable with $u(0)=0, u^{\prime}>0, u^{\prime}(0)=\infty$, $u^{\prime}(\infty)=0, U^{\prime}>0, u^{\prime \prime}<0$, and $U^{\prime \prime} \leq 0$. For simplicity, we set $c(h)=h$, but this is not crucial for any of our results. Let $q^{*} \equiv\left\{q: u^{\prime}\left(q^{*}\right)=1\right\}$, i.e. $q^{*}$ denotes the optimal level of production in any bilateral meeting in the LW market. Also, there exists $X^{*} \in(0, \infty)$ such that $U^{\prime}\left(X^{*}\right)=1$, with $U\left(X^{*}\right)>X^{*}$. Notice that there is no consumption or production in the OTC market. The role of this market is to allow agents to re-balance their portfolios before entering the LW market.

In the first sub-period, all agents consume and produce a general good or fruit. Agents have access to a technology that transforms one unit of labor into one unit of the fruit. In every period a new set of trees are born that produce fruit, as in Lucas (1978). Agents can purchase shares of these trees at the ongoing market price $\psi_{t}$. The supply of these trees is denoted by $A>0$, and it is fixed over time. Each unit of the tree delivers $d$ units of fruit in the next period, and then it dies. Hence, the maturity of the real asset is one period. The second asset that is traded in the $\mathrm{CM}$ is fiat money. The market price of money is denoted by $\varphi_{t}$. Its supply is controlled by a monetary authority, and it evolves according to $M_{t+1}=(1+\mu) M_{t}$, with $\mu>\beta-1$. New money is introduced, or withdrawn if $\mu<0$, via lump-sum transfers to buyers in the $\mathrm{CM}$. Money has no intrinsic value, but it is portable, storable, divisible, and recognizable by all agents. Hence, it can serve as a medium of exchange in the LW market, and help bypass the frictions created by anonymity and the lack of a double coincidence of wants.

After leaving the $\mathrm{CM}$, a measure $\ell<1$ of buyers finds out that they will be able to consume in the forthcoming LW market. We refer to these buyers as the C-types, and to the remaining $1-\ell$ buyers as the $\mathrm{N}$-types. As we discuss below, the only asset that can serve as means of payment in LW is fiat money. Since C-type and N-type buyers are ex-ante identical, the latter hold some cash that they will not use in the current period. This gives rise to potential gains from trade, which can be exploited in the OTC market, as the C-types can readjust their liquidity by selling assets for money, and the $\mathrm{N}$-types are the providers of liquidity. We assume that there exists a matching function $f(\ell, 1-\ell) \leq \min \{\ell, 1-\ell\}$, which brings together C-types and N-types. The function $f$ is homogeneous of degree one and increasing in both arguments. Within each match, the terms of trade are determined through proportional bargaining, following Kalai (1977), and we let $\lambda \in[0,1]$ represent the C-type's bargaining power.

The third sub-period is the standard decentralized market of Lagos and Wright (2005). Ctype buyers meet with sellers in a bilateral fashion, and the two parties negotiate over the quantity of the good produced and the payment. Due to anonymity, trade has to be quid pro 
quo. In contrast with a number of papers in the monetary-search literature, which assume that money and real assets compete as means of payment (see for example Geromichalos et al), here only money can serve as a medium of exchange. This assumption fits one of the goals of the paper, which is to study whether assets can carry liquidity premia even when they do not serve as media of exchange. ${ }^{3}$ Since most of the interesting results of the paper stem from agents' interaction in the OTC, we keep the setup in the LW market as simple as possible. To that end, we assume that all C-types match with a seller, and we choose the measure of sellers to equal $\ell$. Moreover, in any bilateral meeting, the buyer makes a take-it-or-leave-it offer to the seller.

The framework presented here allows us to study asset pricing, and its link to monetary policy, both within traditional, i.e. Walrasian, markets and within OTC markets a la DGP, which have gained a great degree of popularity recently. Recall that the real asset in our model has a maturity length of one period, and it is first traded in the CM. Assuming that the asset is issued in a Walrasian market is a methodological innovation, due to Lagos and Wright (2005), that (together with quasi linearity) gives rise to degenerate asset holding distributions and, hence, ensures tractability. However, in many cases, the issue price of assets is indeed determined in a competitive setting, although, thereafter, the same assets could be traded over-the-counter, as documented by DGP. As an example, W.R. Hambrecht \& Co. persuaded Google to use an Internet-based auction for their initial public offering (IPO), a process now known as an OpenIPO.

\section{Value Functions and Optimal Behavior}

\subsection{Value Functions}

We begin with the description of the value functions in the $C M$. Consider first a buyer who enters this market with money and asset holdings $(m, a)$. The Bellman's equation is given by

$$
\begin{gathered}
W^{B}(m, a)=\max _{X, H, \hat{m}, \hat{a}}\left\{U(X)-H+\mathbb{E}_{i}\left\{\Omega^{i}(\hat{m}, \hat{a})\right\}\right\} \\
\text { s.t. } X+\varphi \hat{m}+\psi \hat{a}=H+\varphi(m+\mu M)+d a,
\end{gathered}
$$

where variables with hats denote next period's choices, and $\mathbb{E}$ denotes the expectations operator. The function $\Omega^{i}$ represents the value function in the OTC market for a buyer of type

\footnotetext{
${ }^{3}$ This assumption also seems to be realistic, since, typically, we do not observe economic agents using assets as media of exchange. However, we do not claim that we adopt this assumption only because of its resemblance to real-world observation. There might be deeper reasons why agents prefer to use money versus assets in order to carry out transactions. For example, Rocheteau (2011) and Lester, Postlewaite, and Wright (2012) consider environments that do not place any restrictions on what objects agents can use as media of exchange. Both papers show that, under asymmetric information, fiat money can endogenously arise as a superior medium of exchange. We believe that studying these questions is very important, but it is beyond the scope of this paper.
} 
$i=\{C, N\}$, to be described in more detail below. It can be easily verified that, at the optimum, $X=X^{*}$. Using this fact and replacing $H$ from the budget constraint into $W^{B}$ yields

$$
W^{B}(m, a)=U\left(X^{*}\right)-X^{*}+\varphi(m+\mu M)+d a+\max _{\hat{m}, \hat{a}}\left\{-\varphi \hat{m}-\psi \hat{a}+\mathbb{E}_{i}\left\{\Omega^{i}(\hat{m}, \hat{a})\right\}\right\} .
$$

As is standard in models that build on Lagos and Wright (2005), the optimal choice of the agent does not depend on the current state (due to the quasi-linearity of $\mathcal{U}$ ), and the $\mathrm{CM}$ value function is linear. This property will greatly simplify the analysis in what follows. Hence, we collect all the terms in (1) that do not depend on the state variables $m, a$ and write

$$
W^{B}(m, a)=\varphi m+d a+\Upsilon^{B},
$$

where the definition of $\Upsilon^{B}$ is obvious.

Next, consider a seller's value function in the CM. Since in this model we assume that $\mu>$ $\beta-1$, the cost of carrying money (the nominal interest rate) is strictly positive, and the sellers will choose not to carry any money as they leave the CM. A similar argument can be used to claim that sellers will also not hold the real asset as they leave the $\mathrm{CM} .{ }^{4}$ Hence, when a seller enters the CM, she can only hold money that she received during trade in last period's LW market, and the CM value function is given by

$$
\begin{gathered}
W^{S}(m)=\max _{X, H}\left\{U(X)-H+V^{S}\right\} \\
\text { s.t. } X=H+\varphi m,
\end{gathered}
$$

where $V^{S}$ denotes the seller's value function in the forthcoming LW market. Sellers also choose $X=X^{*}$, and $W^{S}$ will be linear, since

$$
W^{S}(m)=U\left(X^{*}\right)-X^{*}+\varphi m+V^{S} \equiv \Upsilon^{S}+\varphi m .
$$

After leaving the $\mathrm{CM}$, and before the OTC market opens, buyers learn whether they will have a chance to access this period's LW market (C-types) or not (N-types). This chance will occur with probability $\ell \in(0,1)$, and by the law of large numbers, a proportion $\ell$ of buyers will be of type $\mathrm{C}$. Thus, the expected value for a buyer with portfolio $(m, a)$, before she enters the OTC market is given by

$$
\mathbb{E}_{i}\left\{\Omega^{i}(m, a)\right\}=\ell \Omega^{C}(m, a)+(1-\ell) \Omega^{N}(m, a) .
$$

\footnotetext{
${ }^{4}$ A careful proof of the result that sellers do not hold any money can be found in Rocheteau and Wright (2005). Regarding the optimal choice of real assets, as we shall see in what follows, there exist equilibria in which the cost of holding the real asset is zero. In this case, the seller is indifferent between holding zero or some positive amount of the asset. However, if we assume that there is a fixed cost, $c>0$, of participating in the Walrasian assets' market, then holding zero assets is the unique optimal choice for the seller, even for an infinitesimally small $c$.
} 
In the OTC market, C-type buyers (who may want additional money) are matched with $\mathrm{N}$-type buyers (who want to mitigate the inflation tax on their money holdings) through the function $f(\ell, 1-\ell)$. Given $f$, we can define the matching probabilities (or arrival rates) for the two types: $a_{C} \equiv f(\ell, 1-\ell) / \ell$, and $a_{N} \equiv f(\ell, 1-\ell) /(1-\ell)$. For consistency with subsequent notation, let $\chi$ denote the units of asset that the C-type transfers to the $\mathrm{N}$-type, and $\psi_{I}$ the monetary price, per unit of asset, at which this transfer takes place. Thus, $\chi \psi_{I}$ stands for the total units of money received by the C-type. These terms will be determined through bargaining in Section 3.3. For now, it is understood that $\chi$ and $\psi_{I}$ will, in general, be functions of the money and asset holdings of both the C-type and the N-type within a match. Let $\tilde{m}$ and $\tilde{a}$ denote the amounts of money and asset that a buyer expects a potential counterparty to carry. Then,

$$
\begin{aligned}
& \Omega^{C}(m, a)=a_{C} V^{B}\left(m+\chi \psi_{I}, a-\chi\right)+\left(1-a_{C}\right) V^{B}(m, a), \\
& \Omega^{N}(m, a)=a_{N} \beta W^{B}\left(m-\chi \psi_{I}, a+\chi\right)+\left(1-a_{N}\right) \beta W^{B}(m, a),
\end{aligned}
$$

where $V^{B}$ denotes a buyer's value function in the $\mathrm{LW}$, and $\chi=\chi(m, \tilde{m}, a, \tilde{a}), \psi_{I}=\psi_{I}(m, \tilde{m}, a, \tilde{a})$. Notice that N-type buyers proceed directly to next period's CM.

Lastly, consider the value functions in the LW market. Let $q(m)$ denote the quantity of good produced by the seller, and $p(m)$ the total payment, in monetary units, made to the seller, where $m$ is the amount of money the buyer is bringing into the match. These terms will be determined in Section $3.2^{5}$ Then, the LW value function for a buyer who enters that market with portfolio $(m, a)$ is given by

$$
V^{B}(m, a)=u[q(m)]+\beta W^{B}[m-p(m), a],
$$

and the LW value function for a seller (who enters with no money or assets) is given by

$$
V^{S}=-q(m)+\beta W^{S}[p(m)] .
$$

Having established the agents' value functions, we now proceed to the description of the terms of trade in the LW and the OTC markets.

\subsection{Bargaining in the LW Market}

Since the LW market opens last, the terms of trade in this market will serve as an input for studying the determination of the terms of trade in the OTC market. Hence, we proceed by backwards induction and study the bargaining problem in LW first.

\footnotetext{
${ }^{5}$ Although we have not solved the bargaining problem in the LW market yet, it is a well-known fact in the monetary-search literature that the solution will only depend on the buyer's portfolio. Moreover, since here only money can be used as means of payment, the bargaining solution will depend only on the buyer's money holdings.
} 
Consider a meeting between a C-type buyer with money holdings $m$ and real asset holdings $a$, and a seller who, in the beginning of the LW sub-period, holds no money or assets. The two parties bargain over a quantity $q$, to be produced by the seller, and a total payment of $p$ units of money, to be made by the buyer. The buyer makes a take-it-or-leave-it offer, maximizing her surplus subject to the seller's participation constraint and the cash constraint. The bargaining problem can be described by

$$
\begin{gathered}
\max _{p, q}\left\{u(q)+\beta W^{B}(m-p, a)-\beta W^{B}(m, a)\right\} \\
\text { s.t. }-q+\beta W^{S}(p)-\beta W^{S}(0)=0,
\end{gathered}
$$

and the cash constraint $p \leq m$. Substituting the value functions $W^{B}, W^{S}$ from (2) and (3) into the expressions above, allows us to re-write the bargaining problem as

$$
\begin{gathered}
\max _{p, q}\{u(q)-\beta \hat{\varphi} p\} \\
\text { s.t. } q=\beta \hat{\varphi} p,
\end{gathered}
$$

and $p \leq m$. Notice that the relevant value of money is $\hat{\varphi}$, because the forthcoming CM opens in the next period. The solution to the bargaining problem is described in the following lemma.

Lemma 1. Define the amount of money that, given tomorrow's price $\hat{\varphi}$, allows the buyer to purchase $q^{*}$

$$
m^{*}=\frac{q^{*}}{\beta \hat{\varphi}} .
$$

Then, the solution to the bargaining problem is given by

$$
\begin{aligned}
& p(m)= \begin{cases}m^{*}, & \text { if } m \geq m^{*}, \\
m, & \text { if } m \leq m^{*} .\end{cases} \\
& q(m)= \begin{cases}q^{*}, & \text { if } m \geq m^{*}, \\
\beta \hat{\varphi} m, & \text { if } m \leq m^{*} .\end{cases}
\end{aligned}
$$

Proof. See the appendix.

As the buyer has all the bargaining power, there is no hold-up problem and the solution to the bargaining problem is straightforward. The only variable that affects the solution is the buyer's money holdings. As long as the buyer carries $m^{*}$ or more, the first-best quantity $q^{*}$ will always be exchanged. On the other hand, if $m<m^{*}$, the buyer does not have enough cash to induce the seller to produce $q^{*}$. The cash constrained buyer will give up all her money, $p(m)=m$, and the seller will produce the quantity of good that satisfies her participation constraint under $p(m)=m$, namely, $q=\beta \hat{\varphi} m$. 


\subsection{Bargaining in the OTC market}

We now study the terms of trade in the OTC market. Although buyers are ex ante identical, only some of them (C-types) will get an opportunity to consume in the forthcoming LW market, and may be willing to trade assets for money. The N-type buyers cannot access the LW in this period and have no use for money (other than as a store of value), so they may be willing to trade money for assets. Hence, although the OTC is an asset market, all trades are driven by agents' different valuations for money. In this way, we formalize DGP's suggestion that asset trade is generated by the varying needs for liquidity of market participants.

Consider a meeting in the OTC between a C-type with money and asset holdings $(m, a)$, respectively, and an N-type with portfolio $(\tilde{m}, \tilde{a})$. Let $\chi$ represent the amount of assets that are transferred from the C-type to the $\mathrm{N}$-type (in principle, this variable may be negative), and let $\psi_{I}$ denote the monetary price per unit of asset that is paid to the seller of assets. Following Kalai's solution, and letting $\lambda \in[0,1]$ denote the C-type's bargaining power, we can write the bargaining problem as

$$
\begin{gathered}
\max _{\chi, \psi_{I}}\left\{V^{B}\left(m+\chi \psi_{I}, a-\chi\right)-V^{B}(m, a)\right\} \\
\text { s.t. } V^{B}\left(m+\chi \psi_{I}, a-\chi\right)-V^{B}(m, a)=\frac{\lambda}{1-\lambda}\left[\beta W^{B}\left(\tilde{m}-\chi \psi_{I}, \tilde{a}+\chi\right)-\beta W^{B}(\tilde{m}, \tilde{a})\right],
\end{gathered}
$$

and the cash and asset constraints $\chi \in[-\tilde{a}, a]$, and $\chi \psi_{I} \in[-m, \tilde{m}]$.

This problem has a very intuitive explanation. With proportional bargaining, the objective is to maximize the C-type's surplus, under the constraint that the $\mathrm{N}$-type's payment equals a fixed proportion of that surplus (specifically, $(1-\lambda) / \lambda$ ). Since the C-type has a consumption opportunity, she will proceed to the LW market (where her value function is given by $V^{B}$ ) with an additional $\chi \psi_{I}$ units of money, but also with asset holdings reduced by the amount $\chi$. On the other hand, the N-type will proceed directly to the next period's $\mathrm{CM}$, hence the value functions $W^{B}$ are discounted by $\beta$. If one substitutes the value functions $W^{B}, V^{B}$ from (2) and (7) into the expression above, the bargaining problem can be re-written as

$$
\begin{gathered}
\max _{\chi, \psi_{I}}\left\{u\left[q\left(m+\chi \psi_{I}\right)\right]-u[q(m)]+\beta\left[\hat{\varphi} \chi \psi_{I}+\hat{\varphi} p(m)-\hat{\varphi} p\left(m+\chi \psi_{I}\right)-d \chi\right]\right\} \\
\text { s.t. } u\left[q\left(m+\chi \psi_{I}\right)\right]-u[q(m)]+\beta\left[\hat{\varphi} \chi \psi_{I}+\hat{\varphi} p(m)-\hat{\varphi} p\left(m+\chi \psi_{I}\right)-d \chi\right]=\frac{\beta \lambda}{1-\lambda}\left(d \chi-\hat{\varphi} \chi \psi_{I}\right)
\end{gathered}
$$

and $\chi \in[-\tilde{a}, a], \chi \psi_{I} \in[-m, \tilde{m}]$. It is understood that the expressions $q(\cdot), p(\cdot)$ are described by the solutions to the LW bargaining problem described earlier.

Notice that if we solve equation (11) with respect to $\beta d \chi$, we obtain

$$
\beta d \chi=\beta \hat{\varphi} \chi \psi_{I}+(1-\lambda)\left\{u\left[q\left(m+\chi \psi_{I}\right)\right]-u[q(m)]+\beta \hat{\varphi}\left[p(m)-p\left(m+\chi \psi_{I}\right)\right]\right\} .
$$


This expression admits a more intuitive interpretation than its original counterpart. It states that the N-type should receive an amount of assets whose discounted value equals the real discounted value of the money she is giving up (the term $\beta \hat{\varphi} \chi \psi_{I}$ ), plus a fraction $1-\lambda$ of the net surplus created when a monetary transfer of $\chi \psi_{I}$ is made to the C-type (the term in curly brackets). ${ }^{6}$ Substituting the term $\beta d \chi$ from (12) into (10), simplifies the bargaining problem to

$$
\max _{\chi, \psi_{I}} \lambda\left\{u\left[q\left(m+\chi \psi_{I}\right)\right]-u[q(m)]+\beta \hat{\varphi}\left[p(m)-p\left(m+\chi \psi_{I}\right)\right]\right\},
$$

subject to the constraint in (11), and the feasibility constraints $\chi \in[-\tilde{a}, a]$, and $\chi \psi_{I} \in[-m, \tilde{m}]$. As is standard with proportional bargaining, the C-type's surplus turns out to be equal to a fraction $\lambda$ (her bargaining power) of the total surplus generated when the $\mathrm{N}$-type transfers to the C-type $\chi \psi_{I}$ units of money in return to the $\chi$ units of the asset. The solution to this bargaining problem is described in the following lemma.

Lemma 2. Consider a meeting in the OTC market between a C-type and an N-type with portfolios $(m, a)$ and $(\tilde{m}, \tilde{a})$, respectively, and define the cutoff level of asset holdings

$$
\bar{a}(m, \tilde{m}) \equiv \begin{cases}\frac{1}{\beta d}\{(1-\lambda)\{u[\beta \hat{\varphi}(m+\tilde{m})]-u(\beta \hat{\varphi} m)\}+\lambda \beta \hat{\varphi} \tilde{m}\}, & \text { if } m+\tilde{m}<m^{*}, \\ \frac{1}{\beta d}\left\{(1-\lambda)\left[u\left(\beta \hat{\varphi} m^{*}\right)-u(\beta \hat{\varphi} m)\right]+\lambda \beta \hat{\varphi}\left(m^{*}-m\right)\right\}, & \text { if } m+\tilde{m} \geq m^{*} .\end{cases}
$$

Then the solution to the bargaining problem is given by

$$
\begin{aligned}
\chi(m, \tilde{m}, a) & = \begin{cases}\bar{a}(m, \tilde{m}), & \text { if } a \geq \bar{a}(m, \tilde{m}), \\
a, & \text { if } a<\bar{a}(m, \tilde{m}) .\end{cases} \\
\psi_{I}(m, \tilde{m}, a) & = \begin{cases}\frac{\min \left\{m^{*}-m, \tilde{m}\right\}}{\bar{a}(m, \tilde{m})}, & \text { if } a \geq \bar{a}(m, \tilde{m}), \\
\psi_{I}^{a}(m, a), & \text { if } a<\bar{a}(m, \tilde{m}),\end{cases}
\end{aligned}
$$

where $\psi_{I}^{a}(m, a)$ solves

$$
(1-\lambda)\left\{u\left[\beta \hat{\varphi}\left(m+a \psi_{I}^{a}\right)\right]-u(\beta \hat{\varphi} m)\right\}+\lambda \beta \hat{\varphi} a \psi_{I}^{a}=\beta d a .
$$

Proof. See the appendix.

Lemma 2 has a very intuitive interpretation. If $\tilde{m} \geq m^{*}-m$, the C-type should receive $m^{*}-m$, which will allow her to purchase $q^{*}$ in the forthcoming LW market. On the other hand, if $\tilde{m}<m^{*}-m$, the constrained optimal requires the N-type to hand over all her money to the

\footnotetext{
${ }^{6}$ As we shall see in more detail below, how much surplus is created by this transaction depends crucially on the C-type's original money holdings $m$, i.e. how far she is from being able to buy $q^{*}$.
} 
C-type. However, one needs to worry about whether the C-type has enough assets to compensate the N-type for these transfers of liquidity. This critical level of assets depends on whether $m+\tilde{m}$ exceeds $m^{*}$ or not, and it is given by the term $\bar{a}(m, \tilde{m})$. If $a \geq \bar{a}(m, \tilde{m})$, the asset constraint does not bind. In this case, the total money transfer is $\chi \psi_{I}=\min \left\{m^{*}-m, \tilde{m}\right\}$, and the C-type gives up exactly $\bar{a}(m, \tilde{m})$ units of the asset. On the other hand, if $a<\bar{a}(m, \tilde{m})$, the C-type gives away all her assets, $\chi=a$, and the per unit price is determined such that the sharing rule of the surplus between the two parties (equation (15)) is satisfied. Notice that the bargaining solution is never affected by the N-type's asset holdings.

It should be noted that the suggested solution assumes that trade in the OTC takes place only if a strictly positive surplus is generated. For example, consider the case in which $m+\tilde{m} \geq$ $m^{*}$, and assume that $a$ is unlimited. Strictly speaking, any $\chi \psi_{I} \geq m^{*}-m$ is part of the bargaining solution. However, when an amount of money $\chi \psi_{I}>m^{*}-m$ changes hands, the C-type and the N-type are just swapping assets for money, and no surplus is generated, since the Ctype can already purchase $q^{*}$ in the LW market. To keep the analysis simple in the forthcoming sections, we assume that in cases like this $\chi \psi_{I}=m^{*}-m$.

Some properties of the bargaining solution are worth emphasizing. In particular, it is interesting to see how the solutions $\chi$ and $\psi_{I}$ depend on the bargaining power parameter, $\lambda$, and how the per unit price $\psi_{I}$ depends on the C-type's money holdings. Clearly, $\chi$ and $\psi_{I}$ depend crucially on the values of $m, \tilde{m}, a$, and there are four cases to consider, depending on whether $m+\tilde{m}$ is greater or smaller than $m^{*}$, and whether $a$ is greater or smaller than $\bar{a}(m, \tilde{m})$. Here, we focus on the case where $m+\tilde{m} \geq m^{*}$, and $a$ is plentiful, i.e. $a \geq \bar{a}(m, \tilde{m})$, but similar arguments can be used to study comparative statics in the remaining cases.

Under this specification, the amount of assets traded in the OTC match is given by

$$
\chi=\frac{(1-\lambda)\left[u\left(q^{*}\right)-u(\beta \hat{\varphi} m)\right]+\lambda\left(q^{*}-\beta \hat{\varphi} m\right)}{\beta d},
$$

and the derivative of this expression with respect to $\lambda$ is

$$
\frac{\partial \chi}{\partial \lambda}=\frac{[u(\beta \hat{\varphi} m)-\beta \hat{\varphi} m]-\left[u\left(q^{*}\right)-q^{*}\right]}{\beta d}<0 .
$$

The last inequality follows from the facts that $\beta \hat{\varphi} m<\beta \hat{\varphi} m^{*}=q^{*}$, and the function $u(q)-q$ is maximized at $q=q^{*}$. This result is very intuitive. As the C-type's bargaining power increases, the amount of assets that she has to give up in order to get the first-best amount of money, $m^{*}-m$, goes down.

Next, consider the OTC price of the asset. When $m+\tilde{m} \geq m^{*}$ and $a \geq \bar{a}(m, \tilde{m})$, we have

$$
\psi_{I}=\frac{m^{*}-m}{(1-\lambda)\left[u\left(q^{*}\right)-u(\beta \hat{\varphi} m)\right]+\lambda\left(q^{*}-\beta \hat{\varphi} m\right)} \beta d .
$$


The derivative of this expression with respect to $\lambda$ is

$$
\frac{\partial \psi_{I}}{\partial \lambda}=\frac{\left[u\left(q^{*}\right)-q^{*}\right]-[u(\beta \hat{\varphi} m)-\beta \hat{\varphi} m]}{\left\{(1-\lambda)\left[u\left(q^{*}\right)-u(\beta \hat{\varphi} m)\right]+\lambda\left(q^{*}-\beta \hat{\varphi} m\right)\right\}^{2}} \beta d>0 .
$$

As expected, when the bargaining power of the C-type goes up, the price that she obtains for every unit of asset sold, also increases. Notice that, when $\lambda=1, \psi_{I}=d / \hat{\varphi}$, or equivalently, $\hat{\varphi} \psi_{I}=d$. In other words, when the C-type makes take-it-or-leave-it offers to the N-type, the price that she will request, in real discounted terms $\left(\psi_{I}\right.$ is multiplied by the real value of money tomorrow), equals the whole dividend of the asset, leaving no surplus to the N-type.

One might also ask how $\psi_{I}$ varies with the money holdings of the buyer. Take the derivative of $\psi_{I}$ with respect to $m$ to obtain

$$
\frac{\partial \psi_{I}}{\partial m}=\frac{u^{\prime}(\beta \hat{\varphi} m)\left(q^{*}-\beta \hat{\varphi} m\right)-\left[u\left(q^{*}\right)-u(\beta \hat{\varphi} m)\right]}{\left\{(1-\lambda)\left[u\left(q^{*}\right)-u(\beta \hat{\varphi} m)\right]+\lambda\left(q^{*}-\beta \hat{\varphi} m\right)\right\}^{2}}(1-\lambda) \beta d>0 .
$$

Clearly, the sign of $\partial \psi_{I} / \partial m$ will coincide with the sign of the numerator on the right-hand side, and we claim that, for all $m \in\left[0, m^{*}\right)$, it is positive. To see this point, define the numerator in the last expression as $N(m)$. Notice the following three facts: First, $N(0)=\infty$. Second, $\lim _{m \rightarrow m^{*}} N(m)=0$. Third, $N^{\prime}(m)=\beta \hat{\varphi} u^{\prime \prime}(\beta \hat{\varphi} m)\left(q^{*}-\beta \hat{\varphi} m\right)<0$, for all $m<m^{*}$. Summing up these three observations, verifies that $N(m)>0$, for all $m \in\left[0, m^{*}\right)$, which implies $\partial \psi_{I} / \partial m>0$. This result is also fairly intuitive. Since $\psi_{I}$ denotes the price at which the C-type sells her assets, the more money she carries, the closer she is to $m^{*}$, and the less willing she will be to accept a low price for her assets. On the contrary, if $m$ is very small, the C-type is more desperate for a monetary transfer by the N-type, and she will be willing to accept a low price for her assets.

Having established the bargaining solutions in the OTC and LW markets, we now procced to derive the objective function of the buyer and describe optimal behavior.

\subsection{Objective Function and Optimal Behavior}

The goal of this subsection is to describe the buyer's optimal choice of $(\hat{m}, \hat{a})$. To that end, substitute (5) and (6) into (4), and lead the emerging expression for $\mathbb{E}_{i} \Omega^{i}$ by one period to obtain

$$
\begin{aligned}
\mathbb{E}_{i}\left\{\Omega^{i}(\hat{m}, \hat{a})\right\} & =f(\ell, 1-\ell) V^{B}\left(\hat{m}+\chi \psi_{I}, \hat{a}-\chi\right) \\
& +[\ell-f(\ell, 1-\ell)] V^{B}(\hat{m}, \hat{a}) \\
& +f(\ell, 1-\ell) \beta W^{B}\left(\hat{m}-\tilde{\chi} \tilde{\psi}_{I}, \hat{a}+\tilde{\chi}\right) \\
& +[1-\ell-f(\ell, 1-\ell)] \beta W^{B}(\hat{m}, \hat{a}) .
\end{aligned}
$$


The four lines of this expression represent the benefit for a buyer who holds a portfolio of $(\hat{m}, \hat{a})$ and turns out to be a matched C-type, an unmatched C-type, a matched N-type, or an unmatched N-type, respectively. It is understood that the expressions $\chi, \psi_{I}, \tilde{\chi}$, and $\tilde{\psi}_{I}$ are described by the solution to the OTC bargaining problem (Lemma 2), and in particular

$$
\begin{aligned}
\chi & =\chi(\hat{m}, \tilde{m}, \hat{a}), \\
\psi_{I} & =\psi_{I}(\hat{m}, \tilde{m}, \hat{a}), \\
\tilde{\chi} & =\chi(\tilde{m}, \hat{m}, \tilde{a}), \\
\tilde{\psi}_{I} & =\psi_{I}(\tilde{m}, \hat{m}, \tilde{a}) .
\end{aligned}
$$

In these expressions, the first argument represents the C-type's money holdings, the second argument represents the N-type's money holdings, and the third argument stands for the Ctype's asset holdings. Terms with tildes represent the buyer's beliefs about her potential counterparty's money and asset holdings in the OTC market.

The next step is to substitute the value functions $W^{B}$ and $V^{B}$ from (2) and (7), respectively, into (17). Insert the emerging expression into (1), and focus on the terms inside the maximum operator of (1) (i.e. ignore the terms that do not affect the choice variables). We will define this expression as $J(\hat{m}, \hat{a})$, and we will refer to it as the buyer's objective function. After some manipulations, one can verify that ${ }^{7}$

$$
\begin{aligned}
J(\hat{m}, \hat{a}) & =-\varphi \hat{m}-\psi \hat{a} \\
& +f\left\{u\left[\beta \hat{\varphi}\left(\hat{m}+\chi \psi_{I}\right)\right]+\beta d(\hat{a}-\chi)\right\} \\
& +(\ell-f)[u(\beta \hat{\varphi} \hat{m})+\beta d \hat{a}] \\
& +f\left[\beta \hat{\varphi}\left(\hat{m}-\tilde{\chi} \tilde{\psi}_{I}\right)+\beta d(\hat{a}+\tilde{\chi})\right] \\
& +(1-\ell-f)[\beta \hat{\varphi} \hat{m}+\beta d \hat{a}],
\end{aligned}
$$

where the first line represents the cost of purchasing $\hat{m}$ units of money and $\hat{a}$ units of the real asset, and the last four lines admit a similar interpretation as equation (17). Also, we use $f$ as a shortcut for $f(\ell, 1-\ell)$.

We can now proceed with the examination of the buyer's optimal choice of $(\hat{m}, \hat{a})$. We will do so for any possible money and asset prices, and for any given beliefs about other buyers' money and asset holdings. We focus on prices that satisfy $\varphi>\beta \hat{\varphi}$, since we know that this will be always true in steady state equilibria with $\mu>\beta-1$. Also, the asset price has to satisfy

\footnotetext{
${ }^{7}$ To arrive at (18), we have used the fact that, in the LW market, we will always be on the binding branch of the bargaining solution, i.e. $p(m)=m$ and $q(m)=\beta \hat{\varphi} m$, regardless of whether the C-type got matched in the OTC or not. An unmatched C-type never enters LW with money holdings greater than $m^{*}$, because the nominal interest rate is always positive. On the other hand, a matched C-type will never hold money in excess of $m^{*}$, because we have ruled out trades in the OTC that do not generate a positive surplus (see discussion after Lemma 2).
} 
$\psi \geq \beta d$, since violation of this condition would generate an infinite demand for the asset. The optimal behavior of the buyer is described in detail in Lemmas 3 and 4 below, and the proofs are relegated to the appendix. However, since the results of these lemmas are crucial for the analysis, we provide here a non-technical description of the buyer's optimal portfolio choice.

The objective function of the buyer depends on the terms $\chi, \psi_{I}, \tilde{\chi}$, and $\tilde{\psi}_{I}$, which, in turn, depend on the the bargaining protocol in the OTC market. Given the buyer's beliefs ( $\tilde{m}, \tilde{a})$, she can end up in different branches of the bargaining solution, depending on her own choices of $(\hat{m}, \hat{a})$. In general, the domain of the objective function can be divided into five regions, arising from three questions: (i) When the C-type and the N-type pool their money in the OTC market, can they achieve the first-best in the LW market? (ii) If I am a C-type, do I carry enough assets to compensate the N-type? (iii) If I am an N-type, do I expect a C-type to carry enough assets to compensate me? These regions are illustrated in Figure 1 and the derivation of the figure is described in detail in the appendix. In what follows, we provide an intuitive explanation of how the OTC terms of trade vary in the different regions. For this discussion, it is important to recall the definition of the asset cutoff term $\bar{a}(\cdot, \cdot)$ from Lemma 2.

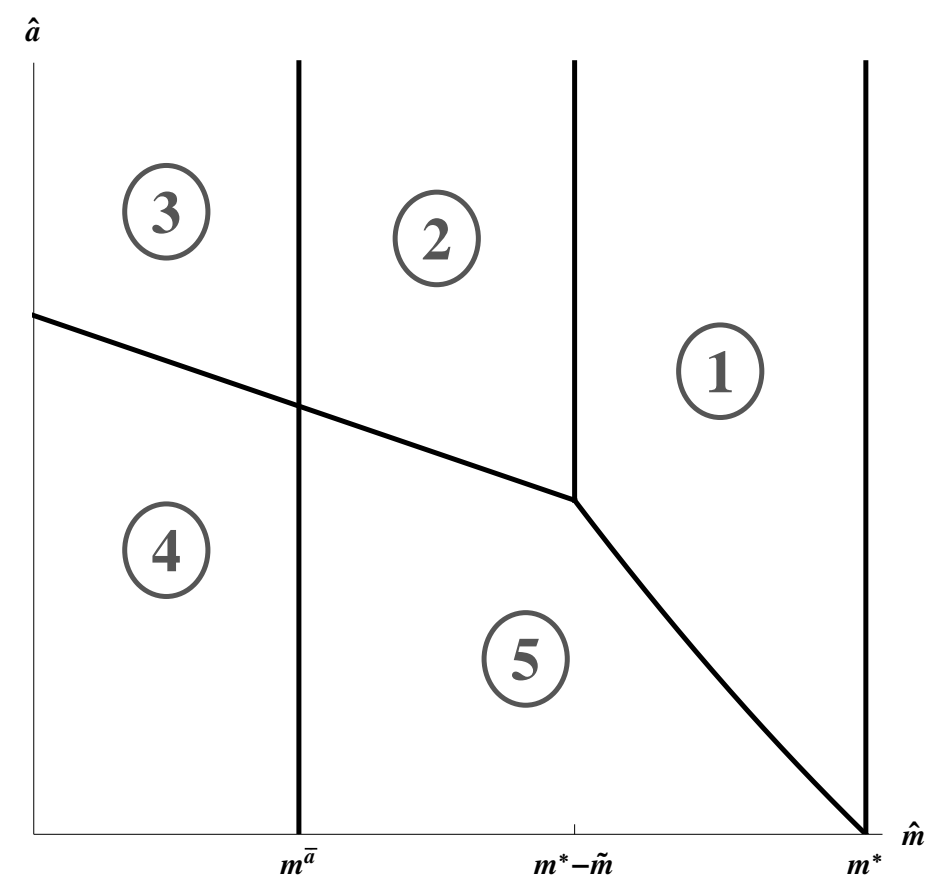

Figure 1: Regions of the individual choice problem.

1. $\hat{m} \in\left(m^{*}-\tilde{m}, m^{*}\right)$ and $\hat{a}>\bar{a}(\hat{m}, \tilde{m})$.

In this region, the money holdings of the C-type and the N-type together allow the C-type to bring the first-best $m^{*}$ into the LW market. If the agent is a C-type, her asset holdings are enough to compensate an $\mathrm{N}$-type for her money. If the agent is an $\mathrm{N}$-type, the potential counterparty may or may not carry enough assets to purchase the first best level of money, 
$m^{*}-\tilde{m}$, but that is a level effect on $J(\hat{m}, \hat{a})$ and does not affect the optimal choice. ${ }^{8}$

2. $\hat{m}<m^{*}-\tilde{m}, \hat{a}>\bar{a}(\hat{m}, \tilde{m})$, but $\tilde{a}<\bar{a}(\tilde{m}, \hat{m})$.

Here there is not enough money in an OTC match to allow the C-type to bring $m^{*}$ into the LW market. If a C-type, the agent carries enough assets to buy all the money of the Ntype, but if an N-type, the agent does not expect the C-type counterparty to carry enough assets to buy all of the agent's money.

3. $\hat{m}<m^{*}-\tilde{m}, \hat{a}>\bar{a}(\hat{m}, \tilde{m})$, and $\tilde{a}>\bar{a}(\tilde{m}, \hat{m})$.

There is not enough money in an OTC match to allow the C-type to bring $m^{*}$ into the LW market. In an OTC match, the agent expects all of the money of the N-type to be traded for less than all of the assets of the C-type (regardless of whether the buyer in question is the $\mathrm{C}$ or the $\mathrm{N}$-type).

4. $\hat{m}<m^{*}-\tilde{m}, \hat{a}<\bar{a}(\hat{m}, \tilde{m})$, but $\tilde{a}>\bar{a}(\tilde{m}, \hat{m})$.

There is not enough money in an OTC match to allow the C-type to bring $m^{*}$ into the LW market. If a C-type, the agent does not carry enough assets to buy all the money of the $\mathrm{N}$-type, but if an N-type, the agent expects the C-type counterparty to carry enough assets to buy all her money.

5. $\hat{a}<\bar{a}(\hat{m}, \tilde{m})$, and either $\tilde{a}<\bar{a}(\tilde{m}, \hat{m})$ or $\hat{m} \in\left(m^{*}-\tilde{m}, m^{*}\right)$.

If a C-type, the agent does not carry enough assets to buy all the money of the N-type. If an N-type, the agent expects not to give away all of her money, either because the C-type counterparty does not carry enough assets to afford it, or because she does not need all of that money. This distinction does not affect the buyer's optimal choice.

Before we proceed to the description of the buyer's optimal choice, we present an auxiliary result that highlights some important properties of the objective function.

Lemma 3. The objective function $J: \mathbb{R}_{+}^{2} \rightarrow \mathbb{R}$ is:

i. continuous everywhere;

ii. differentiable within each of the five regions defined above;

iii. strictly concave in the first argument (money) everywhere;

iv. concave in the second argument (asset), strictly in regions (4) and (5);

\footnotetext{
${ }^{8}$ Since here the objective is to describe the buyer's optimal behavior, we focus on how different choices of $(\hat{m}, \hat{a})$ lead to different branches of the OTC bargaining protocol. In region 1, the buyer is not certain whether her C-type counterparty is asset constrained or not, but she also does not care. What determines region 1 is that within it the buyer's money holdings never affect the terms of trade when she is an N-type. For more details, see the appendix.
} 
v. weakly concave everywhere.

Finally, letting $J^{i}(\hat{m}, \hat{a}), i=1, \ldots, 5$, denote the objective function in region $i$, and $J_{k}^{i}(\hat{m}, \hat{a}), k=1,2$, its derivative with respect to the $k$-th argument, we have:

$$
\begin{aligned}
J_{1}^{1}(\hat{m}, \hat{a}) & =-\varphi+\beta \hat{\varphi}+\beta \hat{\varphi}(\ell-\lambda f)\left[u^{\prime}(\beta \hat{\varphi} \hat{m})-1\right], \\
J_{1}^{2}(\hat{m}, \hat{a}) & =-\varphi+\beta \hat{\varphi}+\beta \hat{\varphi}(\ell-\lambda f)\left[u^{\prime}(\beta \hat{\varphi} \hat{m})-1\right] \\
& +\beta \hat{\varphi} \lambda f\left\{u^{\prime}[\beta \hat{\varphi}(\hat{m}+\tilde{m})]-1\right\} \\
J_{1}^{3}(\hat{m}, \hat{a}) & =-\varphi+\beta \hat{\varphi}+\beta \hat{\varphi}(\ell-\lambda f)\left[u^{\prime}(\beta \hat{\varphi} \hat{m})-1\right] \\
& +\beta \hat{\varphi} f\left\{u^{\prime}[\beta \hat{\varphi}(\hat{m}+\tilde{m})]-1\right\} \\
J_{1}^{4}(\hat{m}, \hat{a}) & =-\varphi+\beta \hat{\varphi}+\beta \hat{\varphi} \ell\left[u^{\prime}(\beta \hat{\varphi} \hat{m})-1\right] \\
& +\beta \hat{\varphi}(1-\lambda) f\left\{u^{\prime}[\beta \hat{\varphi}(\hat{m}+\tilde{m})]-1\right\} \\
& +\beta \hat{\varphi} \lambda f \frac{u^{\prime}\left[\beta \hat{\varphi}\left(\hat{m}+\hat{a} \psi_{I}^{a}(\hat{m}, \hat{a})\right)\right]-u^{\prime}(\beta \hat{\varphi} \hat{m})}{(1-\lambda) u^{\prime}\left[\beta \hat{\varphi}\left(\hat{m}+\hat{a} \psi_{I}^{a}(\hat{m}, \hat{a})\right)\right]+\lambda}, \\
J_{1}^{5}(\hat{m}, \hat{a}) & =-\varphi+\beta \hat{\varphi}+\beta \hat{\varphi} \ell\left[u^{\prime}(\beta \hat{\varphi} \hat{m})-1\right] \\
& +\beta \hat{\varphi} \lambda f \frac{u^{\prime}\left[\beta \hat{\varphi}\left(\hat{m}+\hat{a} \psi_{I}^{a}(\hat{m}, \hat{a})\right)\right]-u^{\prime}(\beta \hat{\varphi} \hat{m})}{(1-\lambda) u^{\prime}\left[\beta \hat{\varphi}\left(\hat{m}+\hat{a} \psi_{I}^{a}(\hat{m}, \hat{a})\right)\right]+\lambda}, \\
J_{2}^{1}(\hat{m}, \hat{a}) & =J_{2}^{2}(\hat{m}, \hat{a})=J_{2}^{3}(\hat{m}, \hat{a})=-\psi+\beta d, \\
J_{2}^{4}(\hat{m}, \hat{a}) & =J_{2}^{5}(\hat{m}, \hat{a})=-\psi \\
& +\beta d\left\{1-f+f \frac{u^{\prime}\left[\beta \hat{\varphi}\left(\hat{m}+\hat{a} \psi_{I}^{a}(\hat{m}, \hat{a})\right)\right]}{(1-\lambda) u^{\prime}\left[\beta \hat{\varphi}\left(\hat{m}+\hat{a} \psi_{I}^{a}(\hat{m}, \hat{a})\right)\right]+\lambda}\right\},
\end{aligned}
$$

where $\psi_{I}^{a}(\cdot, \cdot)$ was defined in (15).

Proof. See the appendix.

The next lemma builds on Lemma 3 and describes in detail the optimal behavior of the representative buyer.

Lemma 4. Taking prices, $(\varphi, \hat{\varphi}, \psi)$, and beliefs, $(\tilde{m}, \tilde{a})$, as given, the optimal choice of the representative buyer, $(\hat{m}, \hat{a})$, satisfies:

a) If the optimal choice is strictly within any region, or on the boundary of region 1 with any other region, it satisfies $\nabla J(\hat{m}, \hat{a})=\mathbf{0}$.

b) If $\varphi>\beta \hat{\varphi}$ and $\psi=\beta d$, the optimal $\hat{m}$ is unique, and any $\hat{a}$ is optimal as long as ( $\hat{m}, \hat{a})$ is in regions 1,2 , or 3 (or on their boundaries). 
c) If $\varphi>\beta \hat{\varphi}$ and $\psi>\beta d$, the optimal choice is unique, and it lies in regions 4 or 5 or on their boundaries, except the boundary of regions 1 and 5 .

Proof. See the appendix.

The technical details of the buyer's optimization problem are relegated to the appendix. Here we highlight the most important properties of the buyer's choice. If the asset price is at the fundamental, i.e. $\psi=\beta d$, the cost of carrying the asset is zero and, therefore, it would be suboptimal for the buyer to be in a region where her assets would not allow her to afford the optimal quantity of money, when a C-type. As a result, when $\psi=\beta d$ the buyer never chooses a portfolio in the interior of regions 4 and 5. If $\psi>\beta d$, carrying the real asset is costly. The optimal choice of the buyer is characterized by the first-order conditions and, graphically, it lies within regions 4 or 5 . For any given prices, the optimal choice of money is uniquely characterized by the first-order condition with respect to $\hat{m}$.

The money demand, $D_{m}$, is plotted in Figure 2 against the ratio $\varphi /(\beta \hat{\varphi})$, which captures the cost of holding money. In this graph, the level of asset holdings is kept fixed at the values $\hat{a}_{1}$ and $\hat{a}_{2}, \hat{a}_{2}>\hat{a}_{1}$. These values are indicated in the lower panel of Figure 2, which replicates Figure 1. Aligning the two plots allows us to easily indicate which region (in terms of Figure 1) the buyer finds herself in, for any choice of $\hat{m}$, given the value of $\hat{a}=\hat{a}_{j}, j=1,2$. The demand for money is, in general, higher under $\hat{a}=\hat{a}_{1}$, because a buyer who holds less assets typically relies more heavily on her own money holdings. In the region where $\hat{m}>\bar{m}^{1-5}, D_{m}^{1}$ and $D_{m}^{2}$ coincide because, for either $\hat{a}=\hat{a}_{1}$ or $\hat{a}=\hat{a}_{2}$, the buyer is in Region 1 , and the marginal benefit of carrying one more unit of money is independent of $\hat{a}$. In particular, in region 1, one additional unit of money: a) serves as a store of value, if the buyer is an N-type; b) allows the buyer to purchase more good in the LW, if she is an unmatched C-type; and c) allows the buyer to reduce her demand for the N-type's money, if she is a matched C-type.

As $\varphi /(\beta \hat{\varphi})$ increases further, the buyer finds herself in region 5 (if $\hat{a}=\hat{a}_{1}$ ) or region 2 (if $\hat{a}=\hat{a}_{2}$ ). In both cases, $D_{m}^{i}$ is continuous, but it is characterized by a kink, and the slope of $D_{m}^{i}$ (in absolute value) is higher on the left side of that kink. To illustrate this property, let $\hat{a}=\hat{a}_{2}$ and consider $D_{m}^{2}$ in a neighborhood of $\bar{m}^{1-2}$, i.e. consider how the marginal benefit of carrying one additional unit of money changes, as the buyer moves from region 1 to region 2. As pointed out earlier, in region 1 an additional unit of money has three effects. The ones indicated by (a) (store of value when N-type) and (b) (higher marginal utility when unmatched C-type) are still relevant as we pass into region 2. But now, when the buyer is a matched C-type, one extra unit of money does not just allow her to lower her demand for the N-type's money (effect (c) above), but, in fact, it allows her to increase her purchasing power in the forthcoming LW market. Hence, for all $\hat{m} \in\left[\bar{m}, \bar{m}^{1-2}\right)$, the demand curve is steeper in comparison to the 

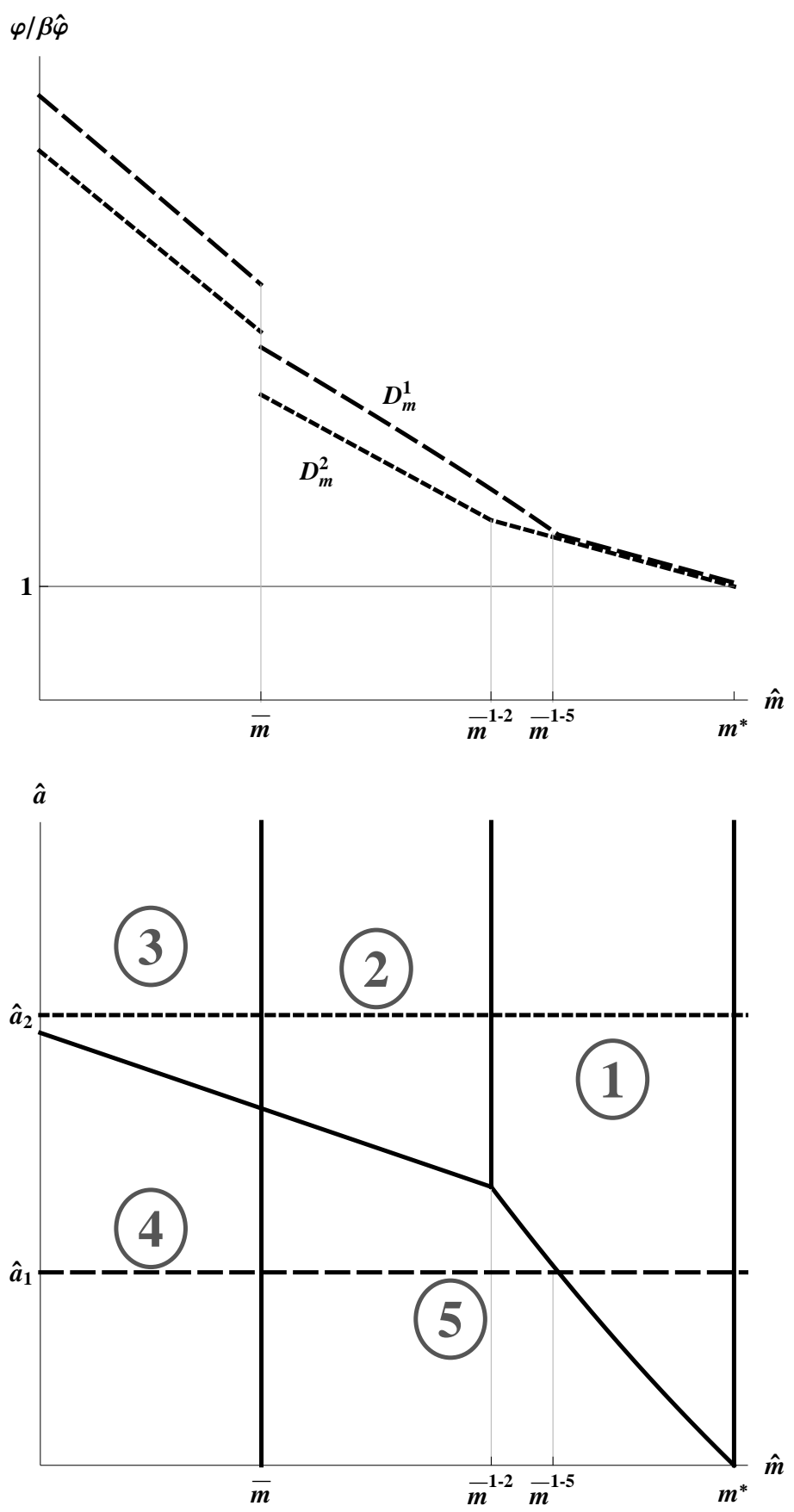

Figure 2: Money demand given high and low asset holdings.

range $\left[\bar{m}^{1-2}, \infty\right)$. Moreover, notice from (19) and (20) that

$$
J_{1}^{2}-J_{1}^{1}=\beta \hat{\varphi} \lambda f\left\{u^{\prime}[\beta \hat{\varphi}(\hat{m}+\tilde{m})]-1\right\} .
$$

This is precisely the term that differentiates $D_{m}^{2}$ on the two sides of $\bar{m}^{1-2}$. Clearly, this term goes to zero when $\hat{m}=\bar{m}^{1-2}=m^{*}-\tilde{m}$. As a result, $D_{m}^{2}$ exhibits a kink but not a jump at $\bar{m}^{1-2}$. The behavior of $D_{m}^{1}$ around $\bar{m}^{1-5}$ admits a similar interpretation.

Finally, the $D_{m}^{i}$ functions exhibit a discontinuity, or a jump, at $\bar{m}$. To illustrate this property, 
again let $\hat{a}=\hat{a}_{2}$ and focus on the behavior of $D_{m}^{2}$ in a neighborhood of $\bar{m}$ (the behavior of $D_{m}^{1}$ around that point can be explained in a similar fashion). Recall from the discussion above that, in region 2, an additional unit of money serves as a store of value, if the buyer is an N-type, and it allows the buyer to purchase more good in the LW, if she is a C-type (matched or unmatched). These effects are also valid as we cross into region 3. However, region 3 is also characterized by a whole new effect, which becomes relevant when the buyer is a matched N-type. In region 3, the C-type counterparty can afford to buy all of the buyer's money, hence the buyer's choice of $\hat{m}$ affects the OTC terms of trade (to the extend that the $\mathrm{N}$-type has some bargaining power, i.e. $\lambda<1$ ). More precisely, the less money the buyer brings, the more desperate the $\mathrm{N}$-type will be for that money and the more assets she will be willing to give up in order acquire it (i.e. $\psi_{I}$ will be very low). In mathematical terms, (20) and (21) imply that

$$
J_{1}^{3}-J_{1}^{2}=\beta \hat{\varphi}(1-\lambda) f\left\{u^{\prime}[\beta \hat{\varphi}(\hat{m}+\tilde{m})]-1\right\} .
$$

Since $\bar{m}<m^{*}-\tilde{m}$, this term is strictly positive when $\hat{m}=\bar{m}$, provided that $\lambda<1$. This gap between the values of $J_{1}^{3}$ and $J_{1}^{2}$ is precisely what leads to the jump of $D_{m}^{2}$ at $\bar{m}$.

Having established the optimal behavior of the representative buyer, we are now ready to discuss equilibrium.

\section{Equilibrium}

In this section we describe equilibrium, with a special focus on the effects of monetary policy on equilibrium asset prices and welfare. We restrict attention to symmetric, steady state equilibria, where all agents choose the same portfolios, and the real variables of the model remain constant over time. ${ }^{9}$ Since, in steady state, the real money balances do not change over time, we have $\varphi M=\hat{\varphi} \hat{M}$, implying that $\varphi / \hat{\varphi}=1+\mu$. Before stating the definition of a steady state equilibrium, it is important to notice that symmetry rules out regions 2 and 4 in Figure 1, since a C-type and an N-type buyer are ex ante identical. On aggregate, only three regions remain:

1. Agents carry enough money and assets so that, when matched in the OTC market, the C-type can acquire sufficient liquidity in order to achieve the first-best in the LW market.

3. Agents carry so little money relative to assets that, when matched in the OTC, the N-type will sell all of her money but the C-type will not achieve the first-best in the LW market.

\footnotetext{
${ }^{9}$ As we established in Section 3, the optimal choice of money holdings is always unique, hence, all agents will choose the same $\hat{m}$. On the other hand, the choice of $\hat{a}$ might not be unique (when $\psi=\beta d$ ). In this case the symmetry assumption is non-trivial.
} 


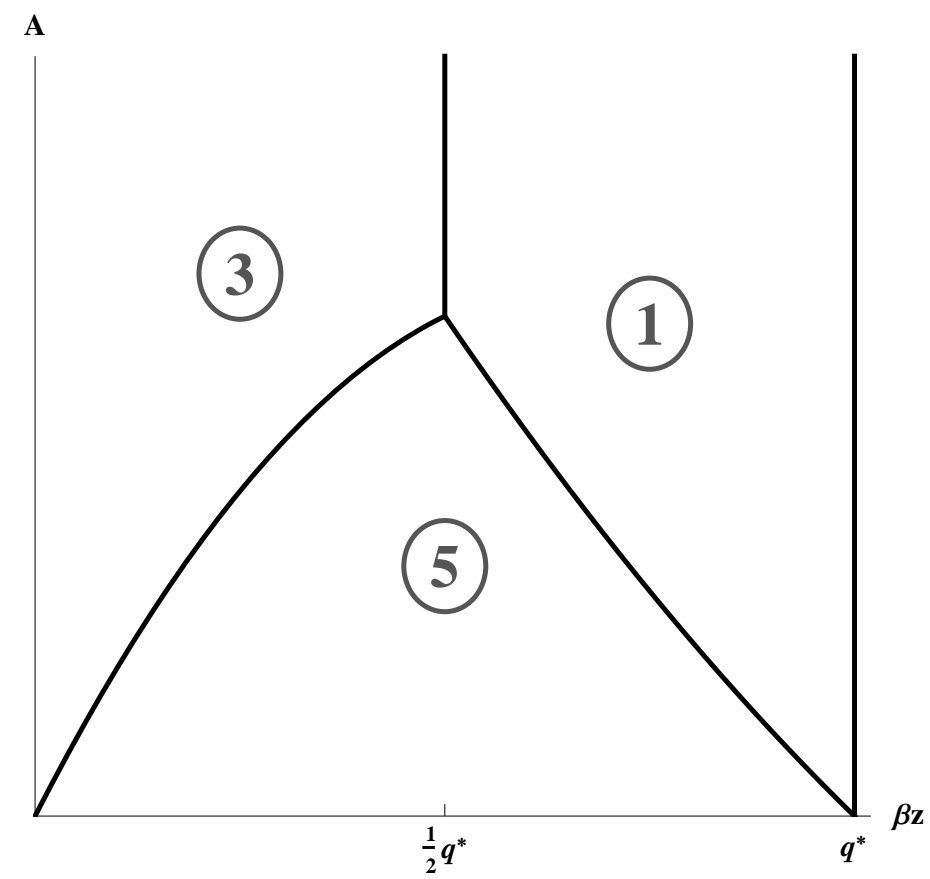

Figure 3: Aggregate regions of equilibrium, in terms of real balances.

5. Agents carry so few assets that, when matched in the OTC, the C-type will sell all of her assets but not obtain enough of the N-type's money in order to achieve the first-best in the LW market.

These regions are described in Figure 3, and we will refer to them as the "aggregate regions", as opposed to the "individual regions" described in Figure 1.

Definition 1. A steady state equilibrium is a list $\left\{\psi, z, q_{1}, q_{2}, \chi, \psi_{I}\right\}$, where $z=\varphi M$ represents the real money balances, $q_{2}$ stands for the amount of good exchanged in the LW market, when the buyer was matched in the preceding OTC market, and $q_{1}$ is the analogous expression for the case of a buyer who was not matched in the OTC. The equilibrium objects are such that:

i. The representative buyer behaves optimally under the equilibrium prices $\psi, \varphi$, and, moreover, $\varphi / \hat{\varphi}=1+\mu$.

ii. The equilibrium quantity $q_{1}$ satisfies $q_{1}=\beta \varphi M=\beta z$, and the quantity $q_{2}$ is defined as the following function of $q_{1}$ :

$$
q_{2}\left(q_{1}\right)= \begin{cases}q^{*}, & \text { in Region 1, } \\ 2 q_{1}, & \text { in Region 3 } \\ \tilde{q}\left(q_{1}\right), & \text { in Region 5 }\end{cases}
$$

where $\tilde{q}$ solves $(1-\lambda)\left[u(\tilde{q})-u\left(q_{1}\right)\right]+\lambda\left(\tilde{q}-q_{1}\right)=\beta d A$. 
iii. The terms of OTC trade $\left(\chi, \psi_{I}\right)$ satisfy (13) and (14).

iv. Markets clear and expectations are rational: $\hat{m}=\tilde{m}=(1+\mu) M$, and $\hat{a}=\tilde{a}=A$.

Lemma 5. A steady state equilibrium $\left\{\psi, z, q_{1}, q_{2}, \chi, \psi_{I}\right\}$ exists and $\left\{z, q_{1}, q_{2}, \chi, \psi_{I}\right\}$ are unique. There exists a region of the parameter space where $\psi$ is indeterminate within a certain range, otherwise $\psi$ is also unique.

Proof. See the appendix.

The definition of equilibrium is straightforward. Notice that the equilibrium quantity of good produced in the LW market depends on whether the buyer was matched in the preceding OTC market or not, since this critically affects her real balances. A buyer who did not match in the OTC, will be able to purchase $q_{1}<q^{*}$ from the seller. How much good the matched (in the OTC) buyer can purchase, depends on whether $q_{1}$ is greater than or smaller than $q^{*} / 2$, and whether the representative buyer has enough assets to purchase the optimal amount of real balances. Of course, in equilibrium, the latter only depends on the exogenous supply, $A$.

Lemma 5 guarantees existence of equilibrium, and states that the equilibrium asset price might not be unique for certain parameter values. We will discuss this issue in detail below. What is perhaps more interesting is to examine how the various equilibrium objects depend on the asset supply, $A$, and the policy parameter, $\mu$, chosen by the authorities. This task is performed in Proposition 1 below, which contains the most important results of the paper. As an intermediate step, it is useful to describe precisely which aggregate region equilibrium will lie in, for different values of $A$ and $\mu$. The following lemma provides the details.

Lemma 6. Define the level of aggregate supply $\bar{A} \equiv\left\{(1-\lambda)\left[u\left(q^{*}\right)-u\left(q^{*} / 2\right)\right]+\lambda q^{*} / 2\right\}(\beta d)^{-1}$.

Case 1: If $A \geq \bar{A}$, then there exists a cutoff $\tilde{\mu}>\beta-1$ such that:

i. If $\mu \in(\beta-1, \tilde{\mu})$, equilibrium is in the interior of region 1;

ii. if $\mu \in(\tilde{\mu}, \infty)$, equilibrium is in the interior of region 3 .

Case 2: If $A<\bar{A}$, then there exist cutoffs $\mu^{\prime}, \mu^{\prime \prime}, \mu^{\prime \prime \prime}$, with $\beta-1<\mu^{\prime}<\mu^{\prime \prime}<\mu^{\prime \prime \prime}$, such that:

i. if $\mu \in\left(\beta-1, \mu^{\prime}\right)$, equilibrium is in the interior of region 1;

ii. if $\mu \in\left(\mu^{\prime}, \mu^{\prime \prime}\right)$, equilibrium is in the interior of region 5;

iii. if $\mu \in\left[\mu^{\prime \prime}, \mu^{\prime \prime \prime}\right]$, equilibrium is on the boundary of regions 5 and 3;

iv. if $\mu \in\left(\mu^{\prime \prime \prime}, \infty\right)$, equilibrium is in the interior of region 3 .

Proof. See the appendix.

Lemma 6 can be explained easily with the assistance of Figure 4. From the analysis of the 


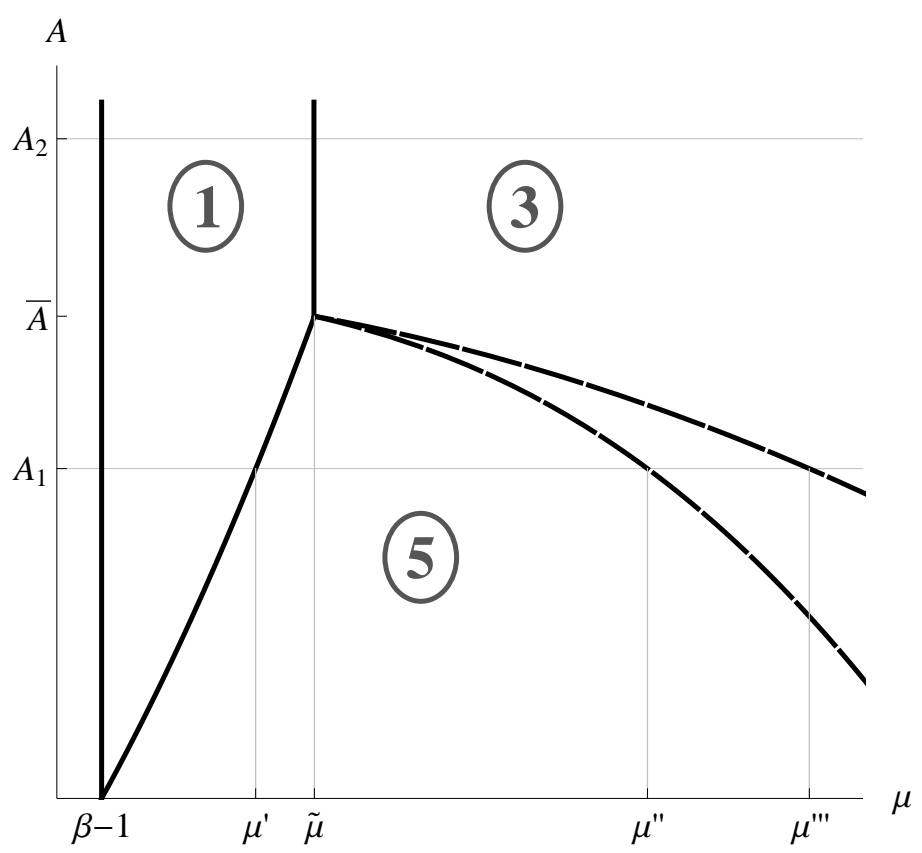

Figure 4: Aggregate regions of equilibrium, in terms of money growth.

buyer's optimal behavior, we know that the demand for money declines as a function of inflation. ${ }^{10}$ If the asset supply is relatively high, more precisely if $A \geq \bar{A}$, rising inflation pushes agents first into aggregate region 1 and then into region 3 . On the other hand, if the asset supply is low, i.e. $A<\bar{A}$, rising inflation pushes agents first into aggregate region 1 , then into region 5 (where the asset is scarce), and finally into region 3 (where the asset is relatively abundant). It is important to keep in mind that, for every $A<\bar{A}$, the region $\left[\mu^{\prime \prime}, \mu^{\prime \prime \prime}\right]$ represents the boundary between aggregate regions 3 and 5, in terms of Figure 3. The reason why this boundary has a positive measure in Figure 4, is the discontinuity of the demand function on this boundary.

We are now ready to state the main results of the paper.

Proposition 1. The properties of the steady state equilibrium are driven by the asset supply. Recall the definition of the cutoff level of asset supply $\bar{A}$ from Lemma 6 . Then:

Case 1: If $A \geq \bar{A}$, then:

a) For any $\mu>\beta-1, \psi=\beta$ d, i.e. the $C M$ asset price is at the fundamental value, and it is not affected by monetary policy;

b) For all $\mu \leq \tilde{\mu}$, the average $L W$ market production is strictly decreasing in $\mu$, unless $f(\ell, 1-\ell)=\ell$, in which case it is unaffected by $\mu$;

c) For all $\mu>\tilde{\mu}$, the average $L W$ market production is strictly decreasing in $\mu$;

d) The volume of OTC trade, given by $f \chi$, is strictly increasing for all $\mu<\tilde{\mu}$, and strictly decreasing for all $\mu>\tilde{\mu}$.

\footnotetext{
${ }^{10}$ Recall that in Figure 2, the demand for money is plotted against $\varphi /(\beta \hat{\varphi})$, which in steady state equals $(1+\mu) / \beta$.
} 
Case 2: If $A<\bar{A}$, then:

a) For all $\mu \in\left(\beta-1, \mu^{\prime}\right] \cup\left(\mu^{\prime \prime \prime}, \infty\right), \psi=\beta d$;

b) For all $\mu \in\left(\mu^{\prime}, \mu^{\prime \prime}\right)$, the CM asset price exceeds the fundamental value, and it is a strictly increasing function of $\mu$, i.e. $\psi=\psi(\mu)>\beta d$, with $\psi^{\prime}(\mu)>0$;

c) For all $\mu \in\left[\mu^{\prime \prime}, \mu^{\prime \prime \prime}\right]$, the $C M$ asset price is indeterminate within the range $\left[\beta d, \psi\left(\mu^{\prime \prime}\right)\right]$;

d) For all $\mu \leq \mu^{\prime}$, the average LW market production is strictly decreasing in $\mu$, unless $f(\ell, 1-\ell)=\ell$, in which case it is unaffected by $\mu$;

e) For all $\mu \in\left(\mu^{\prime}, \mu^{\prime \prime}\right) \cup\left(\mu^{\prime \prime \prime}, \infty\right)$, the average LW market production is strictly decreasing in $\mu$;

f) For all $\mu \in\left[\mu^{\prime \prime}, \mu^{\prime \prime \prime}\right]$, the average LW market production is unaffected by $\mu$;

g) The volume of OTC trade, $f \chi$, is strictly increasing for all $\mu<\mu^{\prime}$, constant for all $\mu \in\left[\mu^{\prime}, \mu^{\prime \prime \prime}\right]$, and strictly decreasing for all $\mu>\mu^{\prime \prime \prime}$.

In both cases, the OTC (real) asset price, $\varphi \psi_{I}$, could increase or decrease with higher inflation.

Proof. See the appendix.

\subsection{Discussion of Equilibrium Results}

Proposition 1 reveals that the driving force of equilibrium is the exogenous asset supply. If the asset is plentiful, in the precise sense that $A \geq \bar{A}$, the C-type in every OTC meeting will acquire the optimal amount of liquidity. Whether this liquidity will allow the C-type to purchase $q^{*}$ in the forthcoming LW market or not, depends on whether we are in aggregate region 1 or 3 , which, in turn, depends on whether $\mu$ is smaller or greater than $\tilde{\mu}$. In either case, however, one additional unit of the asset cannot help the agents increase their LW consumption, since the asset constraint is already not binding in the interior of aggregate regions 1 or 3 . As a result, the asset can never be priced higher than its fundamental value, $\psi=\beta d$, and this price will not be affected by changes in the monetary policy (part (a) of Case 1 of the proposition).

If $A \geq \bar{A}$, the inflation rate does affect real balances and, consequently, production in the LW market and welfare. We define the average LW market production as $q_{L W} \equiv(\ell-f) q_{1}+f q_{2}$. The equilibrium real balances and the quantity $q_{1}$ are strictly decreasing in $\mu$ throughout the range of monetary policies. However, $q_{2}$, the amount of good produced in the LW market when the buyer was matched in the preceding OTC, is constant for all $\mu \leq \tilde{\mu}$. This is true because, for this range of inflations, each agent carries real balances that allow her to purchase $q^{*} / 2$, or more, in the LW (on her own). Since the asset is plentiful, in any OTC meeting, the C-type buys from the N-type the exact amount of real balances that allows her to purchase $q^{*}$ in the LW market. Hence, if $f(\ell, 1-\ell)=\ell$, i.e. all C-types match in the OTC, we have $q_{L W}=\ell q_{2}$, which is not affected by $\mu$ (part (b) of Case 1). Finally, if $\mu>\tilde{\mu}$, we are in aggregate region 3 , and by Definition $1, q_{2}=2 q_{1}$. In this case, $q_{L W}=(\ell+f) q_{1}$ and, therefore, it is decreasing in $\mu$, even if every C-type 


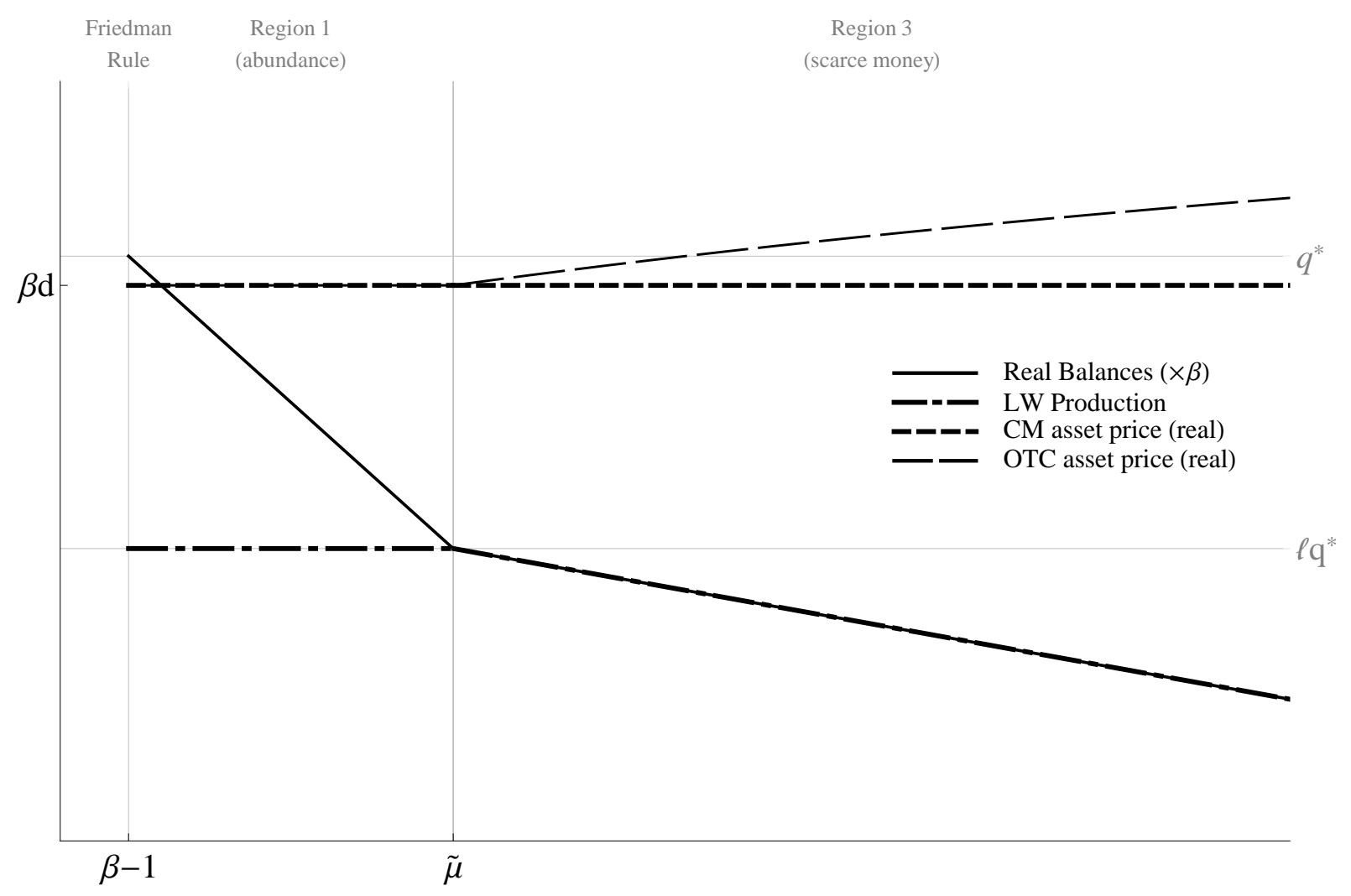

\section{Money growth $\mu$}

Figure 5: Effects of money growth, with perfect matching $(f=\ell)$ and $A \geq \bar{A}$.

gets matched in the OTC (part (c) of Case 1). These results are depicted in Figure 5.

The equilibrium properties of the asset price are richer and more interesting if the asset is relatively scarce. This case is depicted in Figure 6. With $A<\bar{A}$, the levels of inflation $\mu^{\prime}<\mu^{\prime \prime}<\mu^{\prime \prime \prime}$, defined in Lemma 6, become critical. For $\mu \leq \mu^{\prime}$ equilibrium lies in aggregate region 1, and for $\mu>\mu^{\prime \prime \prime}$, it lies in region 3. These regions share a common feature: in both of them, in any OTC meeting, the asset constraint does not bind, i.e. the asset is plentiful. However, the reason that leads to an abundant asset in each of these extreme cases is completely different. In aggregate region 1, the asset is abundant because agents carry a lot of their own money, so they do not need a large transfer of liquidity from their N-type counterparty. As $\mu$ increases, one would think that the C-type would want to acquire more liquidity in the OTC, and, perhaps, her (fixed) asset holdings might not allow her to do so. But if $\mu>\mu^{\prime \prime \prime}$, inflation is so large, and agents carry so little money, that the amount of asset $A$ is in fact enough to purchase all of that money. In a sense, the asset is abundant because real balances are scarce. Regardless of whether we are in aggregate region 1 or 3 , the abundance of the asset in these regions dictates that the $\mathrm{CM}$ asset price cannot exceed the fundamental, i.e. $\psi=\beta d$ (part (a) of Case 2).

For $\mu \in\left(\mu^{\prime}, \mu^{\prime \prime}\right)$, equilibrium lies in aggregate region 5 . In this region the asset is scarce, in the sense that one additional unit of the asset could relax the OTC bargaining constraint, and 


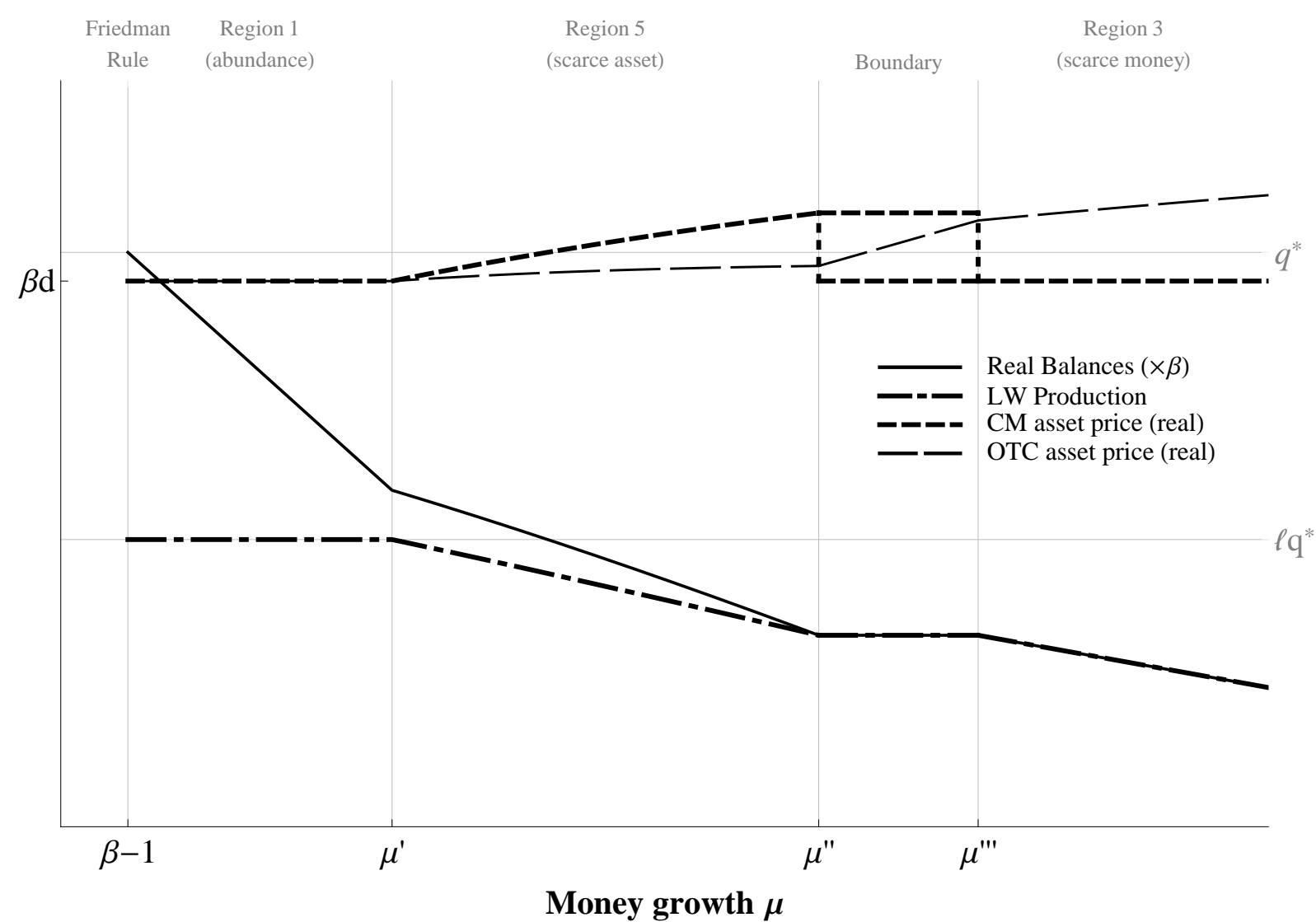

Figure 6: Effects of money growth, with $f=\ell$ and $A<\bar{A}$.

allow the C-type to increase her LW consumption. ${ }^{11}$ Hence, in the parameter space under consideration, the $\mathrm{CM}$ asset price carries a liquidity premium, which reflects the ability of a marginal increase of the asset supply to boost the agent's consumption in the LW market. This result obtains even though the asset does not serve as a medium of exchange or collateral, nor does it help agents enter a repurchase agreement, as discussed in Lagos (2011). In our framework, the asset is sold at a premium because of its property to help agents rebalance their portfolios once the liquidity shocks have been realized.

Now consider the effect of inflation on the asset price for $\mu \in\left(\mu^{\prime}, \mu^{\prime \prime}\right)$. Keeping $A$ fixed and increasing $\mu$ (within region 5) implies that each agent leaves the $\mathrm{CM}$ with less money. Thus, for a C-type who gets matched in the OTC, her valuation of the N-type's money is now larger. Since carrying more assets into the OTC allows the C-type to acquire more of that money, agents are willing (ex ante) to pay a price $\psi$ that (exceeds $\beta d$ but also) is increasing in $\mu$. Summing up, for $\mu \in\left(\mu^{\prime}, \mu^{\prime \prime}\right)$, we have $\psi=\psi(\mu)>\beta d$, and $\psi^{\prime}(\mu)>0$ (part (b) of Case 2).

The positive relationship between the $\mathrm{CM}$ asset price and the inflation rate, within the region $\mu \in\left(\mu^{\prime}, \mu^{\prime \prime}\right)$, resembles the findings of some papers in the related literature, such as Geromichalos et al and Lester et al. However, it should be pointed out that the forces that lead to this

\footnotetext{
${ }^{11}$ Mathematically, this is represented by the second line of $J_{2}^{5}$ in (25).
} 
result are quite different. In the papers mentioned above, fiat money and real assets compete as media of exchange. ${ }^{12}$ An increase in $\mu$ induces agents to substitute money with the real asset, thus increasing its demand and, consequently, its equilibrium price. In our paper, the real asset cannot play the role of money as a medium of exchange in the LW market, but it can help agents increase their liquidity indirectly, when swapped with money in the OTC market. As $\mu$ goes up, the agents' valuation of the asset also rises, because the service that the asset provides, i.e. avoiding the inflation tax, becomes more essential.

Next, consider the region of monetary policies $\mu \in\left[\mu^{\prime \prime}, \mu^{\prime \prime \prime}\right]$. Recall that, by Lemma 6 this region of $\mu$ represents the boundary of regions 3 and 5 in Figure 3, and we know that, at this boundary, the demand for money exhibits a discontinuity. As a result, any change of $\mu$ within this range, leaves equilibrium real balances unaffected. Since $z$ does not move with $\mu$, and since the asset is enough to purchase the optimal amount of real balances in the OTC (we are not in the interior of region 5 anymore), the representative buyer is happy to purchase $A$ units of the asset in the $\mathrm{CM}$, for any price between the fundamental, $\beta d$, and the supremum of the set $\left\{\psi(\mu): \mu \in\left(\mu^{\prime}, \mu^{\prime \prime}\right)\right\}$. This leads to the price indeterminacy result described in part (c) of Case 2 of the proposition.

We now turn to the description of equilibrium production in the LW market under various monetary policies. In general, a higher inflation reduces the equilibrium real balances, with the exception of the region $\left[\mu^{\prime \prime}, \mu^{\prime \prime \prime}\right]$. As we know from previous discussion, an increase in $\mu$ within this region leaves real balances unaffected, and, hence, for any $\mu$ in that region both $q_{1}$ and $q_{2}$ remain constant, and so does the LW production $q_{L W}$ (part (f) of Case 2). For any $\mu \notin\left[\mu^{\prime \prime}, \mu^{\prime \prime \prime}\right]$, the decreasing real balances imply that $q_{1}$ is necessarily decreasing. The quantity $q_{2}$ is also decreasing, with the exception of the region $\left(\beta-1, \mu^{\prime}\right]$. Hence, for any $\mu \notin\left[\mu^{\prime \prime}, \mu^{\prime \prime \prime}\right], q_{L W}=(\ell-f) q_{1}+f q_{2}$, is decreasing in $\mu$, with a potential exception in region $\left(\beta-1, \mu^{\prime}\right]$, when $f(\ell, 1-\ell)=\ell$. In that case, we have $q_{L W}=\ell q_{2}$, which is unaffected by $\mu$ (parts (d) and (e) of Case 2).

One of the most novel results of the paper, discussed in Proposition 1, concerns the effect of monetary policy on the OTC (real) asset price $\varphi \psi_{I}$. Unlike the CM asset price, which is typically non-decreasing in $\mu$, and strictly increasing for certain parameter values $(A<\bar{A}$ and $\left.\mu \in\left(\mu^{\prime}, \mu^{\prime \prime}\right)\right)$, the OTC price could increase or decrease with $\mu$. In principle, a higher inflation rate generates two opposing effects. On the one hand, it strengthens the desire of a (matched) $\mathrm{N}$-type to get rid of her money, which tends to increase the OTC price. On the other hand, since agents leave the CM with less money, the C-type's valuation for her counterparty's money rises, so she is willing to accept a lower price for the asset.

Which of the two forces described above dominates depends on parameter values, specifically, on $\lambda, \ell$, and $f(\ell, 1-\ell)$. The OTC asset price is more likely to be decreasing in $\mu$ when $\lambda$ is low, $\ell$ is low, and $f(\ell, 1-\ell)$ is high. When $\lambda$ is low, the bargaining outcome is closer to the C-

\footnotetext{
${ }^{12}$ In Geromichalos et al, money and real assets are perfect substitutes. Lester et al allow for different degrees of substitutability and eventually endogenize agents' decision on which objects to accept as means of payment.
} 


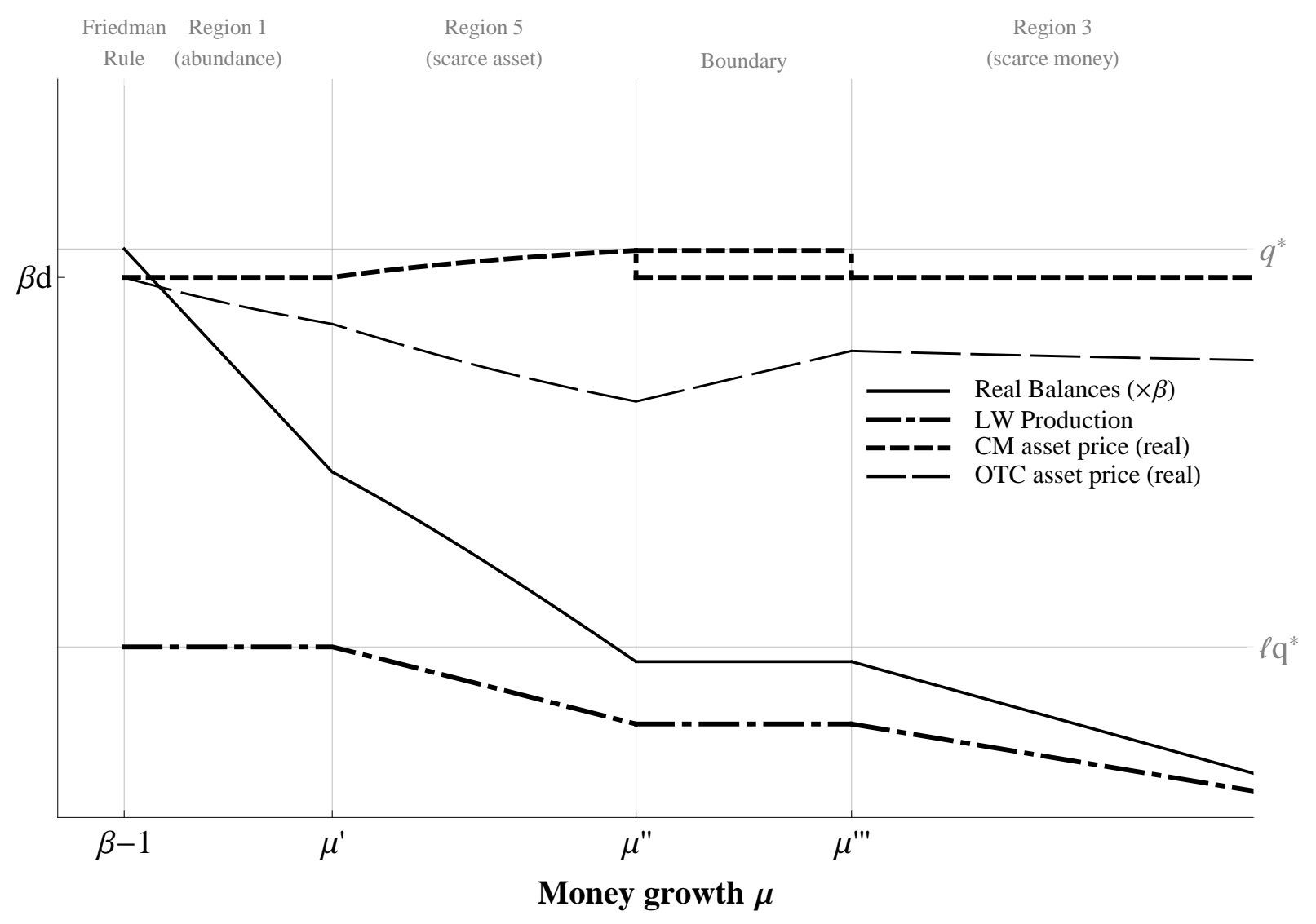

Figure 7: Effects of money growth, with $f=\ell, A<\bar{A}$, and $\lambda$ and $\ell$ low. Unlike in Figure 6, the OTC asset price is mostly decreasing with inflation.

type's outside option, which becomes worse for higher $\mu$, because the C-type is more desperate to obtain extra money. When $\ell$ is low, consumption opportunities are rare, and the incentive to hold money ex-ante is very sensitive to inflation. In a similar fashion, when $f(\ell, 1-\ell)$ is high, agents know that they can obtain more money as needed in the OTC market, therefore money demand is very elastic. In both cases, real balances fall very quickly as $\mu$ increases, which makes the C-type willing to accept a lower price for the asset as she needs to acquire more money in the OTC. Figure 7 depicts a case of low $\lambda$, low $\ell$, and perfect matching $f=\ell$, as an example of how the OTC asset price could be decreasing with inflation.

Finally, it is interesting to study the effect of inflation on the volume of OTC trade, $f \chi$. Figure 8 plots this variable against $\mu$, for two levels of asset supply, $A_{2}>\bar{A}$ (part (d) of Case 1), and $A_{1}<\bar{A}$ (part (g) of Case 2). In both cases, $f \chi$ is hump-shaped, which is a very intuitive result. When inflation is low, agents leave the $\mathrm{CM}$ with a lot of money, thus they need small transfers of liquidity in the OTC, implying that only a small volume of assets will be traded. At the Friedman rule, the OTC market practically closes down because the role of the asset, as a device that helps agents avoid the inflation tax, is completely diminished. As $\mu$ goes up, the role of the OTC is upgraded, and the volume of trade in that market rises as well. Finally, beyond a certain level of $\mu$, the trade volume declines again, not because the need of agents to avoid 


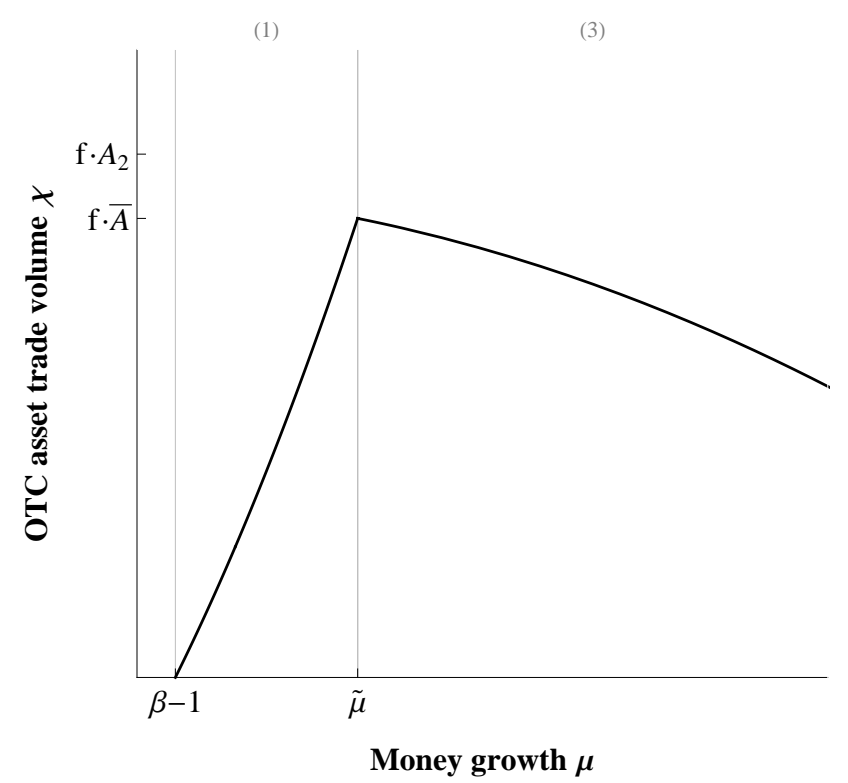

(a) Case 1: $A \geq \bar{A}$

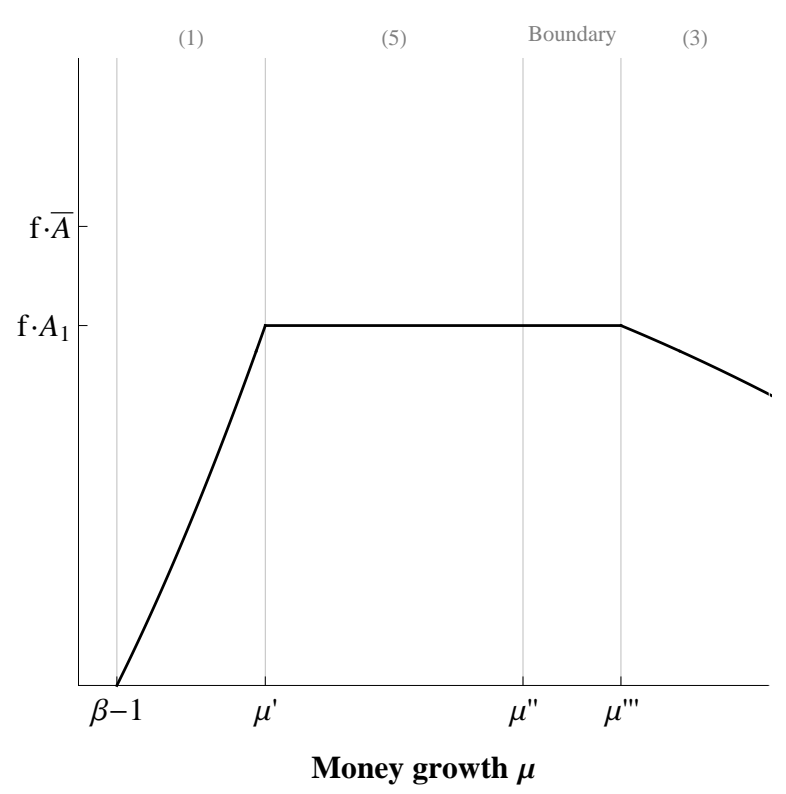

(b) Case 2: $A<\bar{A}$

Figure 8: Trade volume in the OTC market.

the inflation tax is not strong enough (in fact, it is very strong), but because there is not enough liquidity in the OTC to facilitate asset trade (money is scarce). The only difference between the two panels of Figure 8 is that, when $A<\bar{A}, f \chi$ forms a plateau in the region $\left[\mu^{\prime}, \mu^{\prime \prime \prime}\right]$. This result stems from the fact that, for $A<\bar{A}$ and $\mu \in\left[\mu^{\prime}, \mu^{\prime \prime \prime}\right]$, the asset constraint is binding in the OTC, placing an upper bound on the amount of assets that can be traded.

\section{Conclusions}

We revisit a traditional question in monetary economics, the relationship between asset prices and monetary policy. A real asset in fixed supply is issued in a centralized market, a la Lagos and Wright (2005), where agents also choose their money holdings. After the uncertainty regarding consumption in the decentralized market has been resolved, agents may visit a secondary asset market where they can rebalance their positions depending on their liquidity needs. We model this secondary asset market in the style of the OTC markets of Duffie, Gârlea$\mathrm{nu}$, and Pedersen (2005), in which trade is bilateral and agents bargain over the price. This allows us to link asset pricing in OTC markets to monetary policy.

We show that, if the supply of the asset is sufficiently small, the issue price of the asset can include a liquidity premium, reflecting the fact that the asset can help agents avoid the inflation tax. This liquidity premium is increasing in inflation because avoiding the inflation tax becomes more essential. The effect of inflation on the secondary market asset price is ambiguous, and depends on agents' bargaining power in the OTC market, and on how sensitive real balances 
are to inflation. Finally, we consider the effect of inflation on OTC trade. When inflation is low, agents have little need for additional real balances, and when inflation is high, agents have few real balances to trade. Although for different reasons, asset trade is low in both extremes, and hump-shaped as a function of inflation overall.

\section{References}

Berentsen, A., G. Camera, And C. WAller (2007): “Money, credit and banking," Journal of Economic Theory, 135(1), 171-195.

Berentsen, A., And C. WAller (2011): “Outside Versus Inside Bonds: A Modigliani-Miller type result for liquidity constrained economies," Journal of Economic Theory.

BOEL, P., AND G. CAMERA (2006): "Efficient monetary allocations and the illiquidity of bonds," Journal of Monetary Economics, 53(7), 1693-1715.

CHIU, J., AND T. KoEPPL (2011): “Trading dynamics with adverse selection and search: Market freeze, intervention and recovery," .

Duffie, D., N. GÂRleanu, And L. H. Pedersen (2005): “Over-the-Counter Markets," Econometrica, 73(6), 1815-1847.

Geromichalos, A., J. M. LiCARi, And J. SuArez-Lledo (2007): “Monetary Policy and Asset Prices," Review of Economic Dynamics, 10(4), 761-779.

Geromichalos, A., AND I. SimONOVSKA (2011): “Asset Liquidity and International Portfolio Choice," Discussion paper, National Bureau of Economic Research.

JACQUeT, N., AND S. TAN (2010): “The Liquidity Effects of Monetary Policy on Asset Prices," .

KALAI, E. (1977): "Proportional Solutions to Bargaining Situations: Interpersonal Utility Comparisons," Econometrica, 45(7), 1623-30.

KOCHERLAKOTA, N. (2003): "Societal benefits of illiquid bonds," Journal of Economic Theory, 108(2), 179-193.

LAGOS, R. (2008): “The Research Agenda: Liquidity and the Search Theory of Money,” .

LAGOS, R. (2010): "Asset prices and liquidity in an exchange economy," Journal of Monetary Economics, 57(8), 913-930.

(2011): “Asset Prices, Liquidity, and Monetary Policy in an Exchange Economy," Journal of Money, Credit and Banking, 43, 521-552. 
LAGOS, R., AND G. Rocheteau (2009): "Liquidity in asset markets with search frictions," Econometrica, 77(2), 403-426.

LAGOS, R., G. RocheteAu, AND P. WeILl (2011): “Crises and liquidity in over-the-counter markets," Journal of Economic Theory.

LAGOS, R., AND R. WRIGHT (2005): “A Unified Framework for Monetary Theory and Policy Analysis," Journal of Political Economy, 113(3), 463-484.

Lester, B., A. Postlewaite, And R. Wright (2012): "Information, Liquidity, Asset Prices, and Monetary Policy," Review of Economic Studies, forthcoming.

LI, Y., AND Y. LI (2010): “Liquidity, Asset Prices, and Credit Constraints,” Discussion paper, working paper.

LuCAS, Robert E, J. (1978): “Asset Prices in an Exchange Economy,” Econometrica, 46(6), 1429_ 45.

Rocheteau, G. (2011): "Payments and liquidity under adverse selection," Journal of Monetary Economics.

Rocheteau, G., AND R. WRIGHT (2005): "Money in search equilibrium, in competitive equilibrium, and in competitive search equilibrium," Econometrica, 73(1), 175-202.

TOBIN, J. (1965): "Money and economic growth," Econometrica: Journal of the Econometric Society, pp. 671-684.

VAYANOS, D., AND P. WEILl (2006): "A search-based theory of the on-the-run phenomenon," Discussion paper, National Bureau of Economic Research. 


\section{A Appendix}

Proof. Proof of Lemma 1.

The proof is fairly standard. If we substitute $p$ from the the seller's particiaption constraint into the objective function and the cash constraint, we can re-write the bargaining problem as

$$
\max _{q, p}\{u(q)-q\}
$$

subject to $q=\beta \hat{\varphi} p$, and $q \leq \beta \hat{\varphi} m$. As long as $m \geq m^{*}, q$ will be equal to the first-best quantity, i.e. $q=q^{*}$. On the other hand, if $m<m^{*}$, the cash constraint is binding. The buyer will give up all her money, $p(m)=m$, and the quantity produced will be given by the seller's participation constraint, evaluated at $p(m)=m$. In other words, $q=\beta \hat{\varphi} m$.

\section{Proof. Proof of Lemma 2.}

We assume that trade takes place only if a strictly positive surplus is generated. For example, consider the case in which a C-type carries $m \geq m^{*}$. This agent can buy $q^{*}$ in the LW market, and she will also be able to buy $q^{*}$ if she receives more money from the C-type or if she gives up an amount of money up to $m-m^{*}$. In all these cases, the two types are just swapping money for assets, but no surplus is generated. These trivial trades will never affect the equilibrium objects, hence, for simplicity, we rule them out. This, in turn, implies that the only relevant restrictions are $\chi \leq a$ and $\chi \psi_{I} \leq \tilde{m}$, i.e. the C-type will always be a seller of the asset.

From now on, focus on the case where $m, \tilde{m} \leq m^{*}$, which we know will be true in equilibrium, since $\mu>\beta-1$. Since $m \leq m^{*}$, and we have ruled out swapping of money with assets that does not create surplus, we know that the money holdings of the C-type as she enters the LW market will never exceed $m^{*}$. This implies that we will always be on the binding branch of the LW bargaining solution in (8) and (9), i.e. $p(m)=m$, and $q(m)=\beta \hat{\varphi} m$. These observations allow us to re-write the bargaining problem as

$$
\max _{\chi, \psi_{I}} \lambda\left\{u\left[\beta \hat{\varphi}\left(m+\chi \psi_{I}\right)\right]-u(\beta \hat{\varphi} m)-\beta \hat{\varphi} \chi \psi_{I}\right\}
$$

subject to $\chi \leq a, \chi \psi_{I} \leq \tilde{m}$, and (11). The objective function above depends only on the money, $\chi \psi_{I}$, that changes hands, and the only way through which $\chi, \psi_{I}$ are individually relevant is through the feasibility constraints. Defining this objective function as $G\left(\chi \psi_{I}\right)$, we have

$$
\frac{d G}{d\left(\chi \psi_{I}\right)}=\lambda \beta \hat{\varphi}\left\{u^{\prime}\left[\beta \hat{\varphi}\left(m+\chi \psi_{I}\right)\right]-1\right\} \geq 0,
$$

with strict equality for all $\chi \psi_{I}<m^{*}-m$.

The discussion above suggests that the solution to the bargaining problem follows a simple 
algorithm. First, one needs to ask whether the N-type carries enough money such that $m+\tilde{m} \geq$ $m^{*}$. In other words, whether if the two types pool all their money together, the C-type can purchase $q^{*}$ in the LW market. Second, one needs to worry about whether the C-type has enough assets to compensate the N-type for the transfer of liquidity. If $a$ was unlimited, we would always have $\chi \psi_{I}=\min \left\{m^{*}-m, \tilde{m}\right\}$, but the asset constraint may bind. More formally, consider the following cases.

Case 1: $m+\tilde{m} \geq m^{*}$. In this case, if the asset constraint is not binding, we have $\chi \psi_{I}=m^{*}-m$. The critical level of assets that the C-type should carry in order to be able to compensate the $\mathrm{N}$-type for $m^{*}-m$ units of money, can be found by substituting $\chi \psi_{I}=m^{*}-m$ in (11), and solving with respect to $\chi$. If we label this critical level of assets as $\bar{a}(m, \tilde{m})$, we have

$$
\bar{a}(m, \tilde{m})=\frac{1}{\beta d}\left\{(1-\lambda)\left[u\left(\beta \hat{\varphi} m^{*}\right)-u(\beta \hat{\varphi} m)\right]+\lambda \beta \hat{\varphi}\left(m^{*}-m\right)\right\} .
$$

If $a \geq \bar{a}(m, \tilde{m})$, the asset constraint does not bind, and we have $\chi=\bar{a}(m, \tilde{m})$ and $\chi \psi_{I}=m^{*}-m$, implying that $\psi_{I}=\left(m^{*}-m\right) / \bar{a}(m, \tilde{m})$. On the other hand, if $a<\bar{a}(m, \tilde{m})$, the C-type cannot acquire the first-best level of money. In this case, she will give up all her assets, $\chi=a$, and she will pay a price per unit of asset, $\psi_{I}^{a}(m, a)$, which implicitly solves (11), after substituting $\chi=a$. This is just equation (15) in Lemma 2.

Case 2: $m+\tilde{m}<m^{*}$. In this case, if the asset constraint is not binding, we have $\chi \psi_{I}=\tilde{m}$. The critical level of assets that the C-type should carry in order to afford $\tilde{m}$ can be found by substituting $\chi \psi_{I}=\tilde{m}$ in (11), and solving with respect to $\chi$. This yields

$$
\bar{a}(m, \tilde{m})=\frac{1}{\beta d}\{(1-\lambda)\{u[\beta \hat{\varphi}(m+\tilde{m})]-u(\beta \hat{\varphi} m)\}+\lambda \beta \hat{\varphi} \tilde{m}\} .
$$

If $a \geq \bar{a}(m, \tilde{m})$, the asset constraint does not bind, hence $\chi=\bar{a}(m, \tilde{m})$, and $\chi \psi_{I}=\tilde{m}$. If, on the other hand, $a<\bar{a}(m, \tilde{m})$, the C-type gives away all her assets, $\chi=a$, at the per unit price $\psi_{I}^{a}(m, a)$, implicitly defined by (15).

Proof. Derivation of Figure 1.

The various regions of Figure 1 represent different branches of the OTC bargaining solution. On the horizontal axis of that figure we measure the buyer's money holdings in the OTC, $\hat{m}$, and on the vertical axis we measure her real asset holdings, $\hat{a}$. Due to our assumption that $\mu>\beta-1$, the money holdings of the buyer will never satisfy $\hat{m} \geq m^{*}$. Also, in what follows, we will rule out the case in which $\tilde{m} \geq m^{*}{ }^{13}$

The relevant regions arise from the answers to the following three questions:

Question 1: The first question that needs to be answered is whether the sum of the money

\footnotetext{
${ }^{13}$ Strictly speaking, the buyer may not believe that other agents are rational and carry into the OTC an amount of money no greater than $m^{*}$. However, we know that this case will never be relevant in equilibrium. Hence, since we already have to deal with many cases of asset holdings, we choose to ignore this rather trivial region.
} 
holdings of a C-type and an N-type within an OTC match, allows the C-type to achieve the first-best in the LW market. The answer to this question is affirmative, if and only if the buyer's money holdings, $\hat{m}$, exceeds the cutoff level $m^{*}-\tilde{m}$, depicted in Figure 1.

Question 2: The second question that we need to ask is whether the buyer, in the event of being a C-type, carries enough assets to compensate the N-type for the transfer of money that takes place. There are two cases:

Case a: If $\hat{m} \geq m^{*}-\tilde{m}$, we know from Lemma 2, that the amount of assets that C-type needs to carry in order to buy the first best amount of money is given by

$$
\bar{a}=\frac{(1-\lambda)\left[u\left(q^{*}\right)-u(\beta \hat{\varphi} \hat{m})\right]+\lambda \beta \hat{\varphi}\left(m^{*}-\hat{m}\right)}{\beta d} .
$$

The derivative of this term with respect to $\hat{m}$ can be shown to be equal to

$$
\frac{\partial \bar{a}}{\partial \hat{m}}=-\frac{\hat{\varphi}}{d}\left[\lambda+(1-\lambda) u^{\prime}(\beta \hat{\varphi} \hat{m})\right]<0
$$

Case b: If $\hat{m} \leq m^{*}-\tilde{m}$, we know from Lemma 2, that the N-type should hand over to the C-type all her money. The amount of assets that the C-type needs to carry in order to be able to afford this amount of money is given by

$$
\bar{a}=\frac{(1-\lambda)\{u[\beta \hat{\varphi}(\hat{m}+\tilde{m})]-u(\beta \hat{\varphi} \hat{m})\}+\lambda \beta \hat{\varphi} \tilde{m}}{\beta d}
$$

and in this case, we have

$$
\frac{\partial \bar{a}}{\partial \hat{m}}=\frac{(1-\lambda) \hat{\varphi}}{\beta d}\left\{u^{\prime}[\beta \hat{\varphi}(\hat{m}+\tilde{m})]-u^{\prime}(\beta \hat{\varphi} \hat{m})\right\},
$$

which is, clearly, also strictly negative.

Summing up, the C-type carries enough assets to compensate the N-type for the optimal transfer of money, if and only if $\hat{a}$ lies above the curve $\bar{a}(\hat{m}, \tilde{m})$ in Figure 1 . This curve is strictly decreasing for the whole relevant domain of $\hat{m}$, and it is characterized by a kink at $\hat{m}=m^{*}-\tilde{m} .{ }^{14}$ Of course, as $\hat{m} \rightarrow m^{*}, \bar{a}=0$ regardless of $\tilde{m}$, since the C-type can afford $q^{*}$ on her own and does not need to buy any of the N-type's money.

Question 3: The third question that needs to be answered is whether a buyer who turns out to be an N-type, expects the C-type counterparty to carry enough assets to compensate her for her money. In other words, we are interested in whether $\tilde{a} \geq \bar{a}(\tilde{m}, \hat{m})$. Again we distinguish two cases:

\footnotetext{
${ }^{14}$ It can be easily shown that the absolute value of the slope of $\bar{a}$ is lower on the left side of the kink. However, this detail does not play any important role in the analysis.
} 
Case a: If $\hat{m} \geq m^{*}-\tilde{m}$, and the buyer brings one additional unit of money, the amoumt of assets that the N-type needs in order to buy that money does not change. This is because the money that changes hands in this case is given by $m^{*}-\tilde{m}$, which is not affected by $\hat{m}$.

Case b: If $\hat{m} \leq m^{*}-\tilde{m}$, the N-type buyer's money holdings become relevant for the answer to Question 3. Depending on the beliefs $(\tilde{m}, \tilde{a})$, there will exist a threshold of money holdings, denoted by $m^{\bar{a}}$ in Figure 1, such that $\tilde{a} \geq \bar{a}(\tilde{m}, \hat{m})$ if and only if $\hat{m} \leq m^{\bar{a}}$. This threshold is increasing in $\tilde{a}$ and decreasing in $\tilde{m}$. The logic is as follows. For a given $\tilde{m}$, the higher $\tilde{a}$ is, the more money I will need to bring in order to get into the region where my counterparty cannot afford to buy my money. On the other hand, for a given $\tilde{a}$, as $\tilde{m}$ goes up, the C-type counterparty needs less of my money, because she carries more of her own.

How far can the threshold $m^{\bar{a}}$ go? Recall that in the region where $\hat{m} \geq m^{*}-\tilde{m}$, the buyer's money holdings do not affect the term $\bar{a}(\tilde{m}, \hat{m})$. Hence, $m^{\tilde{a}}$ can never lie on the right of the point $m^{*}-\tilde{m}$. As $\tilde{a}$ becomes large, relatively to $\tilde{m}, m^{\bar{a}}$ will exactly coincide with the perpendicular line $m^{*}-\tilde{m}$. It is easy to verify that this will be the case as long as $\tilde{a} \geq \bar{a}\left(\tilde{m}, m^{*}-\tilde{m}\right)$. It is also easy to see that, in this case, the region 2 in Figure 1 ceases to exist.

Notice that the plot in Figure 1 is based on the implicit assumption that $\tilde{a}<\bar{a}\left(\tilde{m}, m^{*}-\tilde{m}\right)$, so that region 2 exists. However, the verbal description of the five regions on page 15 is valid for any set of beliefs $(\tilde{m}, \tilde{a})$. For example, in the description of region 1, we state that "If the agent is an N-type, the potential counterparty may or may not carry enough assets to purchase... $m^{*}-\tilde{m}$, but... (this) does not affect the optimal choice". What we really mean is that it does not matter whether the C-type counterparty carries enough assets or not. What matters, or more precisely, what determines region 1 , is that within this region the buyer's money holdings never affect the terms of trade when she is an N-type. A similar interpretation applies for region 5.

\section{Proof. Proof of Lemma 3.}

Consider first the derivatives of the objective with respect to $\hat{m}$ and $\hat{a}$, i.e. equations (19)-(25). To obtain these conditions we substitute the appropriate solution to the bargaining problem (depending on the region in question) into (18), and we differentiate with respect to $\hat{m}$ or $\hat{a}$.

As an illustration, consider region 2. Recall that in this region, $\hat{m}<m^{*}-\tilde{m}, \hat{a}>\bar{a}(\hat{m}, \tilde{m})$, but $\tilde{a}<\bar{a}(\tilde{m}, \hat{m})$. In words, there is not enough money in an OTC match to allow the C-type to bring $m^{*}$ into the LW market. If a C-type, the agent carries enough assets to buy all the money of the $\mathrm{N}$-type, but if an N-type, the agent does not expect her N-type counterparty to carry enough assets to buy all of her money. Based on this information, we have $\chi=\bar{a}(\hat{m}, \tilde{m}), \chi \psi_{I}=\tilde{m}$, $\tilde{\chi}=\tilde{a}$, and $\tilde{\chi} \tilde{\psi}_{I}=\tilde{a} \psi_{I}^{a}(\tilde{m}, \tilde{a})$. Substituting these terms into the objective, implies that

$$
\begin{aligned}
J^{2}(\hat{m}, \hat{a}) & =-\varphi \hat{m}+(-\psi+\beta d) \hat{a}+f\{u[\beta \hat{\varphi}(\hat{m}+\tilde{m})]-\beta d \bar{a}(\hat{m}, \tilde{m})\} \\
& +(\ell-f) u(\beta \hat{\varphi} \hat{m})+f\left\{\beta \hat{\varphi}\left[\hat{m}-\tilde{a} \psi_{I}^{a}(\tilde{m}, \tilde{a})\right]+\beta d \tilde{a}\right\}+(1-\ell-f) \beta \hat{\varphi} \hat{m} .
\end{aligned}
$$


It is now straightforward to show that $J_{1}^{2}$ and $J_{2}^{2}$ are given by (20) and (24), respectively. The remaining derivations follow exactly the same steps.

Notice that we can solve $J_{1}^{i}=0, i=1, \ldots, 5$, with respect to the term $\varphi /(\beta \hat{\varphi})$, which, in steady state equilibrium, is just one plus the nominal interest rate. This will yield the demand for money as a function of its holding cost. For future reference, it is important to highlight that the money demand is in fact continuous on the boundaries 1-2, and 1-5. ${ }^{15}$ Similarly, we can solve $J_{2}^{i}=0, i=1, \ldots, 5$, with respect to $\psi /(\beta d)$, in order to obtain the demand for the real asset. It can be easily verified that this function is continuous on the boundaries 1-2, 2-5, 2-3, and 4-5.

We now prove the five properties of $J$ stated in Lemma 3.

i. The solution to the OTC bargaining problem is continuous, as can be seen from the proof of Lemma 2. One of the three constraints $\chi \psi_{I} \leq \tilde{m}, \chi \psi_{I} \leq m^{*}-m$, and $\chi \leq a$ must bind, together with equation (11). Each of these is linear in the choice variables. Therefore, $J$ is continuous.

ii. As above, one of the constraints must bind together with equation (11). Each of these is differentiable in the choice variables, and within a region of $J$, the binding constraint does not switch. Furthermore, $J$ is differentiable on those boundaries where both FOCs are continuous (see above).

iii. As $J$ is continuous everywhere and differentiable within each region, $J_{1}$ is defined everywhere except at a finite number of boundary crossings. We need to show that $J_{1}$ is decreasing as a function of $\hat{m}$ within each region, and that $J_{1-} \geq J_{1+}$ on each boundary, where "-" denotes the left derivative and " + " denotes the right derivative.

That $J_{1}$ is strictly decreasing in $\hat{m}$ within regions 1-3 follows immediately from equations (19)(21), and the fact that $u^{\prime}$ is strictly decreasing. In regions 4 and 5 , showing that $J_{1}$ is decreasing in $\hat{m}$ is less obvious. To simplify notation, define $x \equiv \beta \hat{\varphi} \hat{m}$ and $y(x) \equiv \beta \hat{\varphi} \hat{a} \psi_{I}^{a}(\hat{m}, \hat{a})$. In region 5 (where $x+y<q^{*}$ ), we have

$$
J_{1}^{5}=-\varphi+\beta \hat{\varphi}+\beta \hat{\varphi} \ell\left[u^{\prime}(x)-1\right]+\beta \hat{\varphi} \lambda f \frac{u^{\prime}[x+y(x)]-u^{\prime}(x)}{(1-\lambda) u^{\prime}[x+y(x)]+\lambda} .
$$

Since $y(x)$ satisfies (15), applying total differentiation in this equation yields

$$
\frac{d y}{d x}=(1-\lambda) \frac{u^{\prime}(x)-u^{\prime}[x+y(x)]}{(1-\lambda) u^{\prime}[x+y(x)]+\lambda} .
$$

\footnotetext{
${ }^{15}$ The money demand is also continuous on the boundaries of the regions $1-3$ and 4-5 if $\tilde{a} \geq \bar{a}\left(\tilde{m}, m^{*}-\tilde{m}\right)$, in which case region 2 does not exist.
} 
Consequently,

$$
\begin{aligned}
\frac{\partial J_{1}^{5}}{\partial x} & =\frac{\beta \hat{\varphi}}{\left[(1-\lambda) u^{\prime}(x+y)+\lambda\right]^{3}}\left\{f \lambda\left[(1-\lambda) u^{\prime}(x)+\lambda\right]^{2} u^{\prime \prime}(x+y)\right. \\
& \left.+\left[(\ell-f) \lambda+\ell(1-\lambda) u^{\prime}(x+y)\right]\left[(1-\lambda) u^{\prime}(x+y)+\lambda\right]^{2} u^{\prime \prime}(x)\right\} .
\end{aligned}
$$

Since $u^{\prime \prime}(\cdot)<0$, the entire term $\partial J_{1}^{5} / \partial x<0$. In region 4 , the only addition is a term involving $u^{\prime}(\cdot)$, which is clearly decreasing too. Hence, $J_{1}^{4}$ is decreasing in $\hat{m}$ as well.

As we discussed above, $J_{1}$ is continuous across all the boundaries of the various regions, except the boundaries 2-3, 3-4, 4-5, 2-5, and the crossing 2-4. With some algebra, one can check that $J_{1}^{2}<J_{1}^{3}, J_{1}^{3}<J_{1}^{4}, J_{1}^{4}<J_{1}^{5}$, and $J_{1}^{2}<J_{1}^{5}$, across the respective boundaries. Also, $J_{1}^{3}>J_{1}^{5}$ at the crossing 2-3-4-5, establishing the chain $J_{1}^{2}<J_{1}^{5}<J_{1}^{3}<J_{1}^{4}$ at this crossing. Consequently, $J$ is concave in $\hat{m}$ throughout.

iv. As $J$ is continuous everywhere and differentiable within each region, $J_{2}$ is defined everywhere except at a finite number of boundary crossings. We need to show that $J_{2}$ is decreasing as a function of $\hat{a}$ within each region (strictly, in regions 4 and 5), and that $J_{2-} \geq J_{2+}$ on each boundary, where "-" denotes the left derivative and "+" denotes the right derivative.

In regions $1-3, J_{2}^{i}$ is constant, hence weakly concave. We now show that $J_{2}^{i}$ is strictly decreasing in $\hat{a}$ within regions 4 and 5. Applying total differentiation in equation (15), yields

$$
\frac{\partial\left[a \psi_{I}^{a}(m, a)\right]}{\partial a}=\frac{d}{\hat{\varphi}} \frac{1}{(1-\lambda) u^{\prime}\left[\beta \hat{\varphi}\left(m+a \psi_{I}^{a}(m, a)\right)\right]+\lambda} .
$$

Since this expression is clearly positive, and $u^{\prime}$ is strictly decreasing, it follows that $\partial J_{2}^{i} / \partial \hat{a}<0$, for $i=4,5$.

Next, using the definitions of the regions, one can see that $J_{2}$ is continuous across the boundary 2-6, but not the boundaries 3-6 or 4-5. The term

$$
\frac{u^{\prime}\left[\beta \hat{\varphi}\left(\hat{m}+\hat{a} \psi_{I}^{a}(\hat{m}, \hat{a})\right)\right]}{(1-\lambda) u^{\prime}\left[\beta \hat{\varphi}\left(\hat{m}+\hat{a} \psi_{I}^{a}(\hat{m}, \hat{a})\right)\right]+\lambda}
$$

is greater than 1 in regions 5 and 6, because

$$
\hat{m}+\hat{a} \psi_{I}^{a}(\hat{m}, \hat{a})<\min \left\{\hat{m}+\tilde{m}, m^{*}\right\} .
$$

and therefore $u^{\prime}(\cdot)>1$.

v. We need to show that $J_{2}$ is non-increasing as a function of $\hat{m}$ within each region, and across boundaries. First, $J_{2}$ depends on $\hat{m}$ only in regions 5 and 6 . There, the term $\zeta \equiv$ $\beta \hat{\varphi}\left[\hat{m}+\hat{a} \psi_{I}^{a}(\hat{m}, \hat{a})\right]$ is strictly increasing in $\hat{m}$, therefore $u^{\prime}(\zeta)$ is strictly decreasing, and so is $u^{\prime}(\zeta)\left[(1-\lambda) u^{\prime}(\zeta)+\lambda\right]^{-1}$. 
Now, the only boundaries where $J_{2}$ is not a continuous function of $\hat{m}$ are the boundaries of regions 4 and 5, and 3 and 6, which are downward sloping in $(\hat{m}, \hat{a})$-space. On these boundaries, $J_{2-}>J_{2+}$ (see part (iv.)). This is sufficient because an infinitesimal increase in $\hat{m}$ has the same effect as an infinitesimal increase in $\hat{a}$ (the definition of $J_{2+}$ ), and vice versa, when the boundaries are downward sloping.

We conclude that $J_{2}$ is weakly decreasing as a function of $\hat{m}$, therefore $J$ is submodular (money and asset are strategic substitutes). As $J$ is also weakly concave in each argument, it is weakly concave overall.

Proof. Proof of Lemma 4.

a) The fact that $\nabla J(\hat{m}, \hat{a})=\mathbf{0}$, follows from the fact that $J$ is weakly concave overall and differentiable within each region. So if the optimal choice $(\hat{m}, \hat{a})$ is within a region, the firstorder conditions must hold.

b) The fact that $\psi=\beta d$ rules out regions 4 and 5 . To see this point, notice from (25), that for any $(\hat{m}, \hat{a})$ in the interior of these regions, $\psi=\beta d$ implies $J_{2}^{i}>0$, for $i=4,5$. In regions 1-3, money demand is strictly decreasing, so the $\hat{m}$ satisfying $\varphi>\beta \hat{\varphi}$ is unique. But any $\hat{a}$ in regions 1-3 satisfies $J_{2}^{i}=0, i=1,2,3$.

c) The fact that $\psi>\beta d$ rules out the interior of regions 1-3 or the boundary 1-5. To see why, notice from (24), that for any $(\hat{m}, \hat{a})$ in the regions in question, $\psi>\beta d$ implies $J_{2}^{i}<0$, for $i=1,2,3$.

\section{Proof. Proof of Lemma 5.}

The equilibrium objects $q_{1}, q_{2}, \chi$ and $\psi_{I}$ are all deterministic functions of $z$, so it is sufficient to focus on $z$ and $\psi$ alone. Since $\mu>\beta-1$, we have $\varphi>\beta \hat{\varphi}$, and, consequently, parts (b) and (c) of Lemma 4 apply, and an optimal $(\hat{m}, \hat{a})$ exists and $\hat{m}$ is unique. The object $\hat{\varphi}$ (and a proportional $\varphi=(1+\mu) \hat{\varphi})$ must be chosen such that $\hat{m}=(1+\mu) M$. Finally, $z=(1+\mu) \hat{\varphi} M$, so $z$ exists and is unique, and so are the equilibrium objects $q_{1}, q_{2}, \chi$ and $\psi_{I}$. But so far they are still functions of $\psi$.

Finally, set $\hat{a}=A$. Examine the asset demand function (equations (24) and (25)). It is constant in regions 1 and 3 strictly decreasing in $\hat{a}$ in region 5 (also see the proof of Lemma 3, part iv.), and has a jump at the point where $(z, A)$ is on the aggregate boundary 5-3. If $(z, A)$ lies in the interior of region 5 , then $\psi>\beta d$ is unique. If $(z, A)$ lies in the interior of regions 1 or 3 , or on the aggregate boundary $1-3$, then $\psi=\beta d$ is unique. On the other hand, if the equilibrium $z$ is such that $(z, A)$ is on the aggregate boundary $5-3$, then $\psi$ is not unique. Intuitively, both first-order conditions have a jump, and multiple combinations of $(\mu, \psi)$ are consistent with the same point $(z, A)$.

Proof. Proof of Lemma 6.

The asset supply cutoff point $\bar{A}$ is just given by $\bar{a}\left(m^{*} / 2, m^{*} / 2\right)$, and it represents the highest 
level of asset supply that is consistent with aggregate region 5 (see Figure 3).

Case 1: Let $A \geq \bar{A}$. Any equilibrium must satisfy $\hat{a}=A$ for all agents, whether they turn out to be C-types or N-types. By the definition of the regions, we must be in aggregate regions 1 or 3. Now money demand (equations (19) and (21)) is continuous within each region, and also continuous across the boundary 1-3 (because the boundary is defined by $\hat{m}+\tilde{m}=m^{*}$, hence $\left.u^{\prime}(\hat{m}+\tilde{m})=1\right)$, and finally strictly decreasing over the entire range. Therefore, there exists a $\tilde{\mu}>\beta-1$ such that the statement holds.

Case 2: Let $A<\bar{A}$. Any equilibrium must satisfy $\hat{a}=A$ for all agents, whether they turn out to be $\mathrm{C}$-types or $\mathrm{N}$-types. By the definition of the aggregate regions, we could be in regions 1 (high $z$ ), 5 (intermediate $z$ ), or 3 (low $z$ ). Money demand (equations (19), (23), and (21)) is continuous within regions 1 and 5 , and across the boundary 1-5, and strictly decreasing within both regions. Therefore, there exists a $\mu^{\prime}>\beta-1$ as stated in the lemma.

Next, consider a candidate equilibrium $(z, A)$ on the boundary 3-5. By the buyer's optimal behavior, $J_{1}^{5} \leq 0$ and $J_{1}^{3} \geq 0$ at this point. Both $J_{1}^{5}$ and $J_{1}^{3}$ are strictly decreasing in $\mu$ for a fixed $(z, A)$ (and a fixed $\varphi$ ). Let $\mu^{\prime \prime}$ denote the lowest $\mu$ consistent with $J_{1}^{5} \leq 0$, and $\mu^{\prime \prime \prime}$ the highest $\mu$ consistent with $J_{1}^{3} \geq 0$. As shown in the proof of Lemma $3, J_{1}^{5}<J_{1}^{3}$ at that boundary, so it must be that $\mu^{\prime \prime}<\mu^{\prime \prime \prime}$. Finally, $\mu^{\prime}<\mu^{\prime \prime}$ follows from the fact that money demand is strictly decreasing within region 5.

Proof. Proof of Proposition 1.

Case 1: Let $A \geq \bar{A}$. Then the equilibrium can only be in the aggregate regions 1 or 3 .

a) By equation (24), the only solution to $J_{2}=0$ in regions 1 and 3 is $\psi=\beta d$.

b,c) By equations (19) and (21), and using $\hat{m}=M, z=\varphi M=\hat{\varphi} M /(1+\mu)$, and $\varphi=(1+\mu) \hat{\varphi}$, demand for real balances $z$ is strictly decreasing as a function of $\mu$. The average LW market production is $q^{L W} \equiv f q_{2}+(\ell-f) q_{1}$. By Lemma 6, equilibrium is in region 1 if $\mu<\tilde{\mu}$ and in region 3 otherwise. In region $1, q_{2}=q^{*}$ and $q_{1}=\beta z$, so $q^{L W}$ falls if $z$ does unless $f=\ell$. In region $3, q_{2}=2 \beta z$ and $q_{1}=\beta z$, so $q^{L W}$ falls if $z$ does.

d) The measure of matched agents in the OTC, $f$, is constant, and $\chi=\bar{a}(\hat{m}, \tilde{m})$ in regions 1 and 3. So in aggregate region $1, \chi=\left\{(1-\lambda)\left[u\left(q^{*}\right)-u(\beta z)\right]+\lambda\left(q^{*}-\beta z\right)\right\} /(\beta d)^{-1}$, which is strictly decreasing in $z$, therefore $\chi$ is strictly increasing as a function of $\mu$. In aggregate region 3 , on the other hand, $\chi=\{(1-\lambda)[u(2 \beta z)-u(\beta z)]+\lambda \beta z\} /(\beta d)^{-1}$, which is strictly increasing in $z$, therefore $\chi$ is strictly decreasing as a function of $\mu$.

Case 2: Let $A<\bar{A}$. By Lemma 6 , the equilibrium is in regions 1,3 , or 5 , or on the boundary of regions 5 and 3 , for the according money growth rate $\mu$.

a) The range $\mu \in\left(\beta-1, \mu^{\prime}\right]$ corresponds to region 1 , and $\mu \in\left(\mu^{\prime \prime \prime}, \infty\right)$ corresponds to region 3. As shown for Case $1, \psi=\beta d$ in these regions.

b) The range $\mu \in\left(\mu^{\prime}, \mu^{\prime \prime}\right)$ corresponds to region 5 . Asset demand in region 5 satisfies equation (25). As the argument of $u^{\prime}\left[\beta \hat{\varphi}\left(\hat{m}+\hat{a} \psi_{I}^{a}(\hat{m}, \hat{a})\right)\right]$ is less than $m^{*}$ by the definition of the 
region, $\psi>\beta d$. Furthermore, higher inflation reduces $\hat{m}$. The term $\psi_{I}^{a}(\hat{m}, \hat{a})$ is itself an increasing function of $\hat{m}$, as shown in equation (16), so higher inflation reduces the argument and increases the term $u^{\prime}\left[\beta \hat{\varphi}\left(\hat{m}+\hat{a} \psi_{I}^{a}(\hat{m}, \hat{a})\right)\right]$, and also increases $\psi$.

c) The range $\mu \in\left[\mu^{\prime \prime}, \mu^{\prime \prime \prime}\right]$ corresponds to the boundary of regions 5 and 3 , where the objective function has a kink. Clearly, $\psi$ still cannot be lower than $\beta d$. The upper limit of $\psi$ is given by the $\psi\left(\mu^{\prime \prime}\right)$, which is the limit of $\psi(\mu)$ in region 5, because any $\psi>\psi\left(\mu^{\prime \prime}\right)$ would lead the agent to demand less assets than the point on the boundary, and equilibrium would move into the interior of region 5 .

$\mathrm{d}$,e) The proof is the same as for Case 1, parts (b), (c), with the addition of noting that $\hat{m}$ (and hence $z$ ) is a strictly decreasing function of inflation in region 5 as well.

f) The boundary of regions 5 and 3 , together with the fact that $A$ is given, fixes $z$ and hence $q^{L W}=f \cdot 2 \beta z+(\ell-f) \cdot \beta z$.

g) As in part (d) of Case $1, f$, is constant. That $\chi$ is increasing in region 1 and decreasing in region 3 was proven for Case 1, part (d). In region 5 and on the boundary 5-3, the OTC asset constraint is binding, implying that $\chi=A$, which is constant.

Finally, consider the OTC (real) asset price, $\varphi \psi_{I}$. This term can increase or decrease with inflation, and this depends on $\ell, \lambda$, and the shape of the utility function. (The exception is the boundary 5-3, i.e. $\mu \in\left[\mu^{\prime \prime}, \mu^{\prime \prime \prime}\right]$. There, $\varphi \psi_{I}$ must be increasing, because $z$ is constant and the only thing that matters is that the value of money falls with higher $\mu$.) An example is illustrated in Figure 7. 